\title{
Characterizing the Performance of an Image-Based Recognizer for Planar Mechanical Linkages in Textbook Graphics and Hand-Drawn Sketches
}

\author{
Matthew Eicholtz, Levent Burak Kara* \\ Department of Mechanical Engineering, Carnegie Mellon University, 5000 Forbes Avenue, Pittsburgh, PA 15213, USA
}

\begin{abstract}
In this work, we present a computational framework for automatically generating kinematic models of planar mechanical linkages from raw images. The hallmark of our approach is a novel combination of supervised learning methods for detecting mechanical parts (e.g. joints, rigid bodies) with the optimizing power of a multiobjective evolutionary algorithm, which concurrently maximizes image consistency and mechanical feasibility. A rigorous set of experiments was conducted to systematically evaluate the performance of each phase in our framework, comparing various combinations of joint and body detection schemes and feasibility constraints. Precision-recall curves are used to assess object detection performance. For the optimization, in addition to standard accuracy measures such as top- $N$ accuracy, we introduce a new performance metric called user effort ratio that quantifies the amount of user interaction required to correct an inaccurate optimization solution. Current state-of-the-art performance is achieved with (i) one (or a cascade of) support vector machines for joint detection, (ii) foreground extraction to reduce false positives, (iii) supervised body detection using normalized geodesic time, distance, and detected joint confidence, and (iv) feasibility constraints derived from graph theory. The proposed framework generalizes moderately well from textbook graphics to hand-drawn sketches, and user effort ratio results demonstrate the potential power of an interactive system in which simple user interactions complement computer recognition for fast kinematic modeling.
\end{abstract}

Keywords: computer vision, evolutionary multiobjective optimization, image processing, kinematic modeling, object recognition, sketch recognition

\section{Introduction}

A planar mechanical linkage is an assembly of rigid bodies s connected by kinematic pairs (or joints) that constrain its mo4 tion within a plane. With applications in robotics [6, 7], health5 care $[29,38]$, transportation [58, 65], and industrial equipment $6[32,46]$, among others, we observe and make use of the dy7 namic behavior of complex mechanical systems on a daily ba8 sis. Visualizing the coordination motion of mechanical linkages $\ominus$ is indeed a valuable skill for improving design intuition [18], 10 yet during the design and analysis of such dynamic assemblies, ${ }_{11}$ the visual content is largely static in nature, as illustrated in ${ }_{12}$ Figure 1.

${ }_{13}$ To overcome this information deficit, students and engineers 14 may use mental simulations to infer mechanical behavior [36], 15 but this can be difficult for complex problems [35] or for in${ }_{16}$ dividuals with low spatial ability [37]. They also frequently 17 use hand-drawn sketches to convey design ideas, perhaps in18 corporating key annotations and arrows to demonstrate motion; ${ }_{19}$ even so, the burden for visualization remains with the user. Al${ }_{20}$ ternatively, computer simulations can be generated using spe${ }_{21}$ cialized software [1-4], in which users manually create kine${ }_{22}$ matic models. However, this task is often too time-consuming

\footnotetext{
${ }^{*}$ Corresponding author.

Email addresses: meicholt@andrew.cmu .edu (Matthew Eicholtz), lkara@cmu .edu (Levent Burak Kara)
}

a

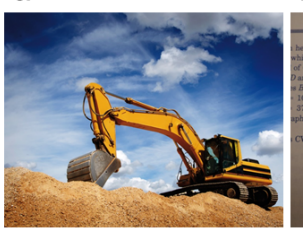
b

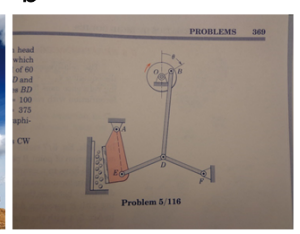

C

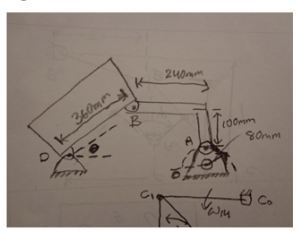

Figure 1: Example mechanical linkages (a) in the real world, (b) in textbooks, and (c) in hand-drawn sketches ${ }^{2}$. The latter two cases represent valid inputs for the recognition framework presented in this paper.

${ }_{23}$ to be practical (e.g. students solving a dynamics homework ${ }_{24}$ problem, professional engineers brainstorming potential design 25 concepts) and may require advanced programming skills, hin${ }_{26}$ dering novice users. There is a clear need for better software ${ }_{27}$ tools that facilitate quick computer-based kinematic visualiza${ }_{28}$ tion of mechanical linkages, filling the gap between ineffective 29 mental simulations and impractical manual model creation.

In this paper, we address that need by proposing a computa${ }_{31}$ tional framework that automatically recognizes the underlying ${ }_{32}$ mechanical structure in images of textbook graphics or hand-

\footnotetext{
${ }^{2}$ Ironically, even the excavator shown here is static in this printed document, despite our usual observation of its dynamic behavior in the real world. Images courtesy of (a) MOBA (www.moba.de), (b) the MECH135 dataset, and (c) the MECHS250 dataset.
} 
${ }_{3}$ drawn sketches. In order to generate a proper kinematic simu34 lation of a planar mechanical linkage, the user typically needs ${ }_{35}$ to specify the number and position of rigid bodies that make up ${ }_{36}$ the linkage, as well as the type and location of joints that dictate 37 relative motion between rigid bodies. The goal of our method 38 is to offload this burden to the computer. We accomplish this ${ }_{39}$ in multiple stages using a joint-centric approach, meaning that ${ }_{40}$ linkages are viewed as a collection of connected joints (as op${ }_{41}$ posed to connected bodies), and each pairwise joint connection 42 indicates that those two joints exist on the same rigid body. In 43 this way, rigid bodies can be inferred from the connections be${ }_{44}$ tween joints. This reduces the problem to localizing joints in ${ }_{45}$ the image, predicting which joints coexist on rigid bodies, and ${ }_{46}$ resolving discrepancies to form reasonable mechanical assem47 blies. For simplicity, we limit our study to planar mechanisms 48 comprising only revolute (pin) joints.

\section{${ }_{49}$ 2. Background}

50 Our computational approach relies on ideas from many disci${ }_{51}$ plines, including computer vision, sketch recognition, and evo${ }_{52}$ lutionary computation. Here, we outline key references in these ${ }_{53}$ areas that influenced the design of our recognition framework.

\section{${ }_{54}$ 2.1. Scene understanding}

${ }_{55}$ The task of identifying structured objects in images is not a 56 new one $[14,24,25]$. Practical applications include face recog57 nition [26, 66], pose estimation [64], and 3D surface estimation 58 [55]. The key difference, though, between previous work in this 59 area and our present domain is that planar mechanisms do not 60 have well-defined structural or spatial dependencies. For exam${ }_{61}$ ple, in face recognition, it is straightforward to learn that a chin 62 should not be located above the nose or that eyes should exist ${ }_{63}$ between the ears; with mechanical linkages, it is less clear if ${ }_{64}$ a specific joint should be systematically connected to another. ${ }_{65}$ Little knowledge is gained about the likelihood of other objects ${ }_{66}$ in the image just from knowing one object's location.

67 Within the current domain of interest, Sato et al. [57] pro${ }_{68}$ posed a vision-based approach for automatically estimating the 69 location of an axis of rotation in a mechanical linkage. The pri70 mary differences between that work and the research in this pa71 per are twofold. First and foremost, it relies on motion tracking 72 from a series of images to capture the moving parts, whereas ${ }_{73}$ our work is restricted to a single image. Second, it seems to 74 be limited to simple mechanisms with only one axis of rota75 tion (single pin joint), whereas our approach can handle more 76 complex kinematic behavior (multiple pin joints).

\section{2.2. Sketch recognition}

78 Two important aspects of sketch recognition that relate to 79 the present work are representation and complexity. With re80 gard to representation, two classes of techniques have emerged 81 in the literature. Stroke-based methods treat each sketch as a 82 sequence of time-stamped strokes, each containing a series of ${ }_{83}$ sample points in space. While some works share similarities ${ }_{84}$ to our domain $[17,33,34,42,52]$, stroke-based methods are
${ }_{85}$ ill-suited for our recognition framework, which is designed to 86 work on rasterized images. Still, there are interesting parallels; 87 for instance, [34] uses a graph representation to combine low${ }_{88}$ level primitives into high-level shapes using geometrical rules. ${ }_{89}$ We also implement graphs in our recognition pipeline, but in90 stead connect low-level joints to form high-level mechanisms ${ }_{91}$ based (partially) on mechanical feasibility rules.

92 The other class of sketch recognition techniques is image${ }_{93}$ based approaches, including the present work, which neglect 94 temporal information and only consider the spatial layout of ${ }_{95}$ pixels. This poses the additional challenge of grouping rele96 vant pixels, depending on the object being recognized. With ${ }_{97}$ regard to sketch complexity, it is important to distinguish be98 tween isolated symbol recognizers and detecting objects in free99 hand sketches, which is a more challenging problem. The task 100 of symbol recognition can be treated as a template matching 101 problem; some examples of successful approaches in this area 102 include [13, 28, 39, 40, 43, 50]. In some sense, the joint recog103 nition algorithm used here is similar to a sliding window sym104 bol recognizer. However, due to the allowable shape variance 105 of objects in mechanical linkages, we do not use unsupervised 106 part templates and instead learn a discriminative model based 107 on local image features.

${ }_{108}$ Within the current domain of interest, researchers have 109 briefly studied the automatic recognition of mechanical systems 110 from sketch input $[17,27,28]$, but these approaches typically 111 involve clean images, well-defined part templates, and some112 times make use of temporal information to aid recognition. Our 113 approach must generalize well to raw images, which are often 114 noisy, may contain extraneous information from other graphics, 115 and do not always contain well-defined part models.

\section{2.3. Evolutionary multiobjective optimization}

The proposed framework includes an evolutionary optimiza118 tion stage to resolve discrepancies from the vision-based de119 tection of joints and joint connections. There is a growing 120 body of research in the area of multiobjective evolutionary al${ }_{121}$ gorithms (MOEAs), especially in regard to real-world applica122 tions. Many well-known MOEAs are based on Pareto dom123 inance [19, 61, 67], which states that a given solution domi124 nates another solution if it is at least as good on all objectives 125 and better on at least one objective. Arguably the most popu126 lar MOEA of this type and the one used in this present work 127 is the nondominated sorting genetic algorithm (NSGA-II) [19], 128 which has been successful largely due to fast computation of 129 nondominated fronts, preservation of elitist solutions, and lack 130 of a user-specified sharing parameter.

${ }_{131}$ The nondominated sorting genetic algorithm was first in132 troduced almost 20 years ago (NSGA, [61]) and improved 8 133 years later (NSGA-II, [19]). The two identifying characteris134 tics of NSGA-II are nondominated sorting and crowding dis135 tance. Nondominated sorting involves locating the Pareto front ${ }_{136}$ (all nondominated solutions), assigning those solutions a rank ${ }_{137}$ of 1 , and iteratively assigning higher ranks to each Pareto front ${ }_{138}$ level, ignoring all previously detected levels. Crowding dis139 tance measures the local spread of solutions and is used to pre140 serve diversity in the population. These parameters are used 
${ }_{141}$ during the selection process as follows. When sorting a set of 142 solutions, any solution with lower (better) rank goes before so${ }_{143}$ lutions with higher rank. Inevitably, there will be cases when 144 the desired number of survivors cuts through one of the Pareto 145 ranks. In this case, the solutions with that rank are sorted with 146 preference given to higher crowding distances. This algorithm 147 has been shown to have success when the number of objectives ${ }_{148}$ is small, so it should be a suitable approach for the problem 149 presented in this paper.

${ }_{150}$ A number of researchers have applied evolutionary algo${ }_{151}$ rithms to the domain of mechanical linkages [5, 9, 41, 44, 45, $15249]$. However, this line of work is concerned with synthesis of 153 new mechanisms rather than analysis of existing ones. Gen154 erally, the optimization goal is to evolve mechanical linkages 155 (many times with fixed topology) for which a coupler point 156 follows a target trajectory path. Additional constraints on the ${ }_{157}$ parameter space make the problem tractable. By contrast, our 158 optimization goal is to evolve mechanical linkages that are con159 sistent with the visual content in an image and kinematically 160 feasible; no path trajectories are prescribed. While these related 161 works on mechanism synthesis are confined to a well-defined 162 parameter space, our approach must deal with the additional 163 challenges of object recognition.

164 For the present domain, the feasibility of a predicted mech165 anism is governed by mechanical principles. These principles ${ }_{166}$ can be formulated as a series of constraints; in this way, large 167 regions of the search space may become infeasible because one 168 or more of the constraints fail. A critical step in algorithm de${ }_{169}$ sign is determining how to handle such constraints. Constraint 170 handling methods can be broadly categorized into two groups: 171 (i) those that always prefer feasible solutions (hard constraints) $172[12,19]$ and (ii) those that treat constraints as objectives (soft 173 constraints) [62]. We employ the latter method in order to allow 174 infeasible, yet strong, solutions to persist because they may be 175 near the constraint boundaries.

\section{3. Related work and contributions}

177 The current work builds upon two previous attempts to solve 178 the problem of automatic image-based kinematic modeling of 179 mechanical linkages. In our first effort [22], we introduced a ${ }_{180}$ baseline approach comprising a fixed-window sliding object de181 tector to localize probable joints, an unsupervised metric called 182 normalized geodesic time to predict which joints should be con${ }_{183}$ nected to each other, and the NSGA-II algorithm [19] to evolve ${ }_{184}$ a small set of feasible mechanical structures using the vision 185 output.

186 Despite initial promising results, there was room for im${ }_{187}$ provement in each phase of the approach. Indeed, one of the 188 benefits of our framework is that core modules (e.g. joint de189 tection, body detection, structural optimization) can be modi190 fied in isolation without affecting the underlying principles of 191 the approach. With this in mind, in a secondary work [21], we 192 enhanced the joint detection algorithm by incorporating multi193 ple context-based classifiers of increasing window size and im194 plementing a greedy foreground extraction technique. Broadly 195 speaking, these modifications had the effect of increasing the
196 confidence gap between true positives (actual joints in the im197 age) and false positives (incorrectly-identified joints) as well as 198 descreasing the total number of false positives, respectively. In 199 addition, we investigated whether training our system on im200 ages of textbook graphics could transfer well to tests on hand201 drawn sketches and vice versa. The primary contribution of that 202 work was the idea that it may be possible to build a powerful ${ }_{203}$ sketch recognition tool without ever needing a sample sketch 204 for learning.

205 In the present work, we shift our focus to the systematic eval206 uation of each phase in our framework. Previously, quantita207 tive comparisons between algorithm variants were made only at 208 the optimization level. In this paper, we incorporate precision209 recall curves to assess the quality of joint and body detection 210 schemes. Also, the performance metrics previously used to 211 evaluate accuracy of evolved solutions were somewhat coarse 212 that is, a binary indicator of success for a given image may not ${ }_{213}$ fully characterize the quality of the optimization routine. Me214 chanical linkages are complex, with many examples containing 215 ten or more parts. We believe a recognition framework that reg216 ularly identifies $90 \%$ of the required components successfully, 217 for example, is better than one that only identifies $10 \%$ of the 218 required components. To that end, we propose a novel metric ${ }_{219}$ called user effort ratio that expresses the amount of user effort 220 required, on average, to correct a solution provided by our opti221 mization routine. In effect, this metric exemplifies the potential 222 benefit of using our system versus fully manual construction of 223 kinematic models.

In addition to the evaluation goals of this work, we also con225 tinue to improve our algorithms by integrating a supervised 226 method for learning pairwise joint connections and creating 227 more robust optimization constraints on mechanical feasibility. 228 In summary, the significant contributions of this paper beyond 229 previous related work include:

1. Inclusion of a supervised learning approach for body detection.

2. Development of new feasibility constraints for optimization based on graph theory.

3. Introduction of a new performance metric (user effort ratio) for assessing overall solution quality.

4. Systematic evaluation of each stage in the recognition

framework, comparing recent algorithm modifications to all previously reported methods.

\section{${ }_{239}$ 4. Overview}

The proposed methodology for recognizing mechanical link${ }_{241}$ ages in images is illustrated in Figure 2. A database of images 242 with labeled ground truth information - mechanical structure ${ }_{243}$ including joint locations and pairwise connectivity - is required 244 for training. During testing, the input to our algorithm is a sin${ }_{245}$ gle image containing a planar mechanical linkage. Model pa246 rameters are unknown a priori, including the number of joints, 247 number of bodies, topology, and geometric configuration. Al248 though we initially targeted textbook graphics, the framework 249 can be applied to images of hand-drawn sketches without any 


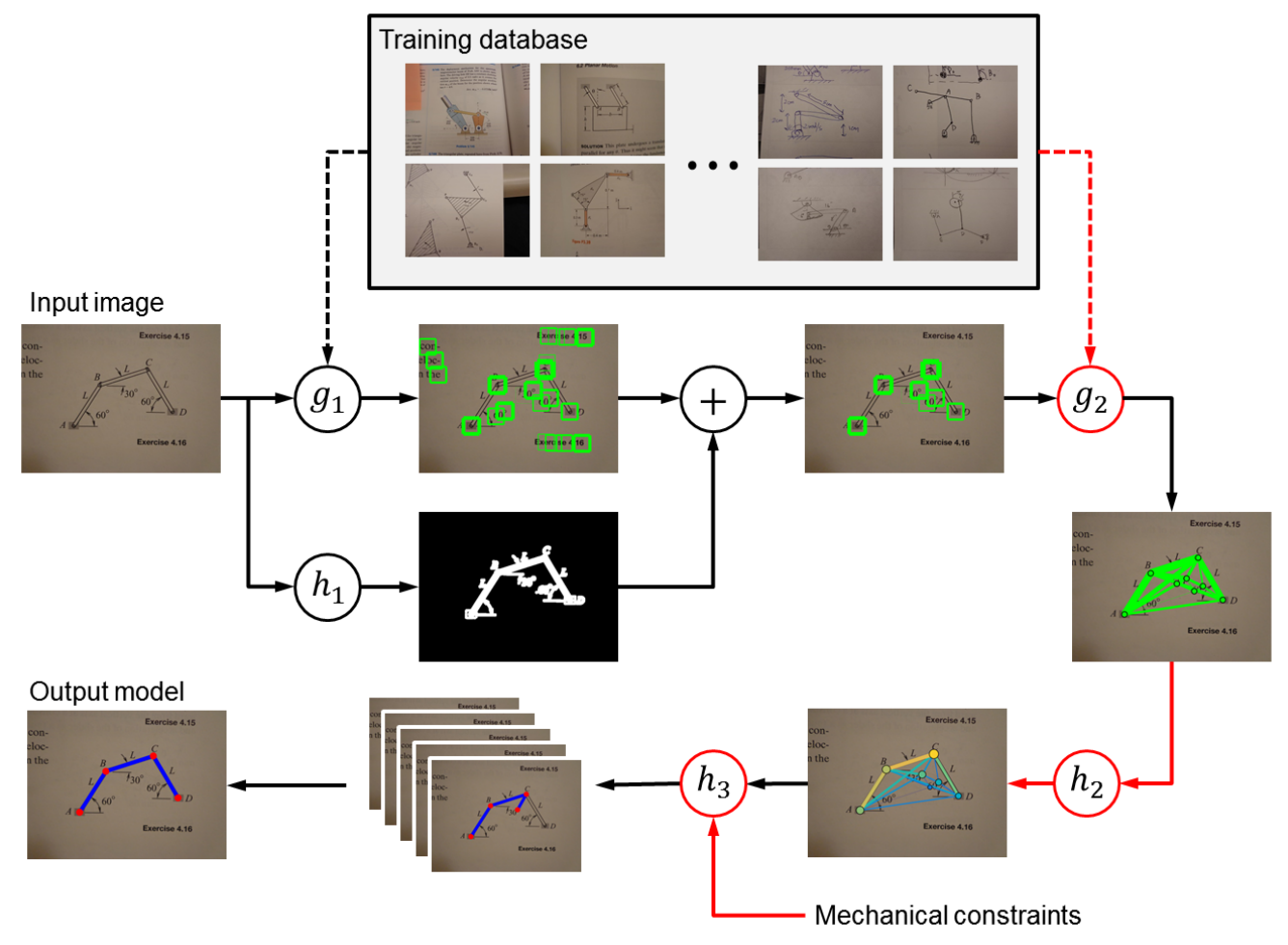

Figure 2: Overview of the proposed recognition framework. Taking a raw image as input, probable joints are detected using a supervised classifier ( $\left.g_{1}\right)$ and filtered using foreground extraction $\left(h_{1}\right)$. Then, rigid bodies are inferred from the supervised detection of pairwise joint connections $\left(g_{2}\right)$, removing any remaining joints that do not have any connections $\left(h_{2}\right)$. The resulting data is passed to an evolutionary algorithm $\left(h_{3}\right)$ that optimizes a graphical model based on fitness criteria related to image consistency and mechanical constraints.

250 modifications. No explicit restrictions are made regarding the 251 position, scale, or orientation of the linkage within the image, 252 although it is assumed the entire mechanism is fully visible. In ${ }_{253}$ addition, images do not need to be pre-processed in any way 254 (e.g. cropping, filtering), so they may contain noise, illumina255 tion changes, and extraneous information such as text, annota256 tions, pencil markings, or partial depictions of other mechanical 257 systems.

258 The fundamental principle of our approach is to identify 259 salient components that make up mechanical linkages first, and 260 then to optimize the collection of detected components into ${ }_{261}$ structural graphs such that results are consistent with visual 262 cues in the image and plausible in terms of engineering mechan263 ics. Mechanical components are detected in a serial manner. ${ }_{264}$ Probable joints are identified first $\left(g_{1}\right)$, with an optional filtering 265 step to ignore background candidates $\left(h_{1}\right)$. Then, the likelihood 266 of body connections between joints is estimated $\left(g_{2}\right)$, followed 267 by a simple filter to remove joints without any connections $\left(h_{2}\right)$. ${ }_{268}$ The optimization $\left(h_{3}\right)$ takes into account the confidence of all 269 vision-based detections as well as mechanical principles to en270 sure that feasible structures are preferred. The output is a small 271 set of kinematic models that can be easily integrated into engi272 neering software packages for further analysis and simulation.

273 In the next section, we discuss the technical details of our 274 algorithms. As stated earlier, portions of the proposed frame275 work were developed in earlier papers [21, 22]. For complete-
276 ness, we describe the entire framework here, but emphasize the 277 components that are unique to this paper, which are identified 278 in Figure 2 by red lines.

\section{5. Technical details}

\section{5.1. Detecting pin joints}

281 The first step in the vision pipeline is to identify the location 282 of joints in an input image. We accomplish this task by running 283 a sliding window over the entire image and classifying each ${ }_{284}$ patch using a linear support vector machine ( $g_{1}$ in Figure 2$)$. 285 The SVM is trained over HOG features [15] computed on a set 286 of example images; this process is outlined in Figure 3.

287 The training data comprises positive and negative image ${ }_{288}$ patches extracted from selected hand-labeled images. Positive 289 samples contain a pin joint, while negative samples do not. The 290 negative patches are randomly selected from the training im291 ages, making sure they do not overlap with positive samples. 292 Similar to Fu and Kara [27], we augment the training set by ap${ }_{293}$ plying a series of simple transformations to each positive exam294 ple: reflection about the vertical or horizontal axis, and rotation 295 by 90,180 , and 270 degrees. This effectively increases the pos296 itive training data by a factor of 5, which likely improves the 297 discriminative power of the joint detector. In addition, we fol298 low the approach of Dalal and Triggs [15] to improve classifi299 cation accuracy by mining hard negatives after initially training 
a

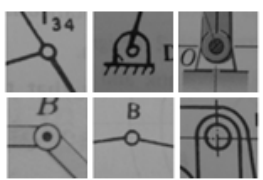

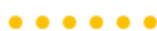

b

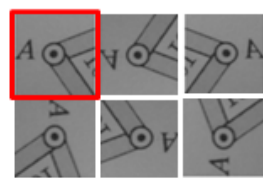

$\bullet \circ \circ \circ$ c

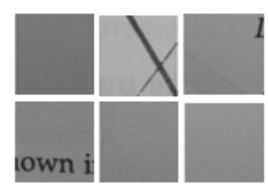

$\bullet \bullet \bullet \bullet \bullet$ d,e

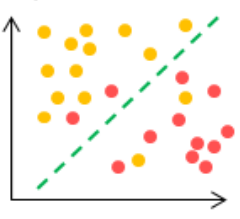

f

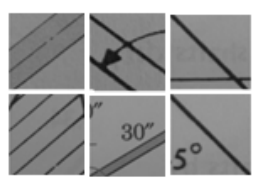

$\bullet \bullet \bullet \bullet \bullet$

Figure 3: Training a support vector machine for joint detection. (a) Extract positive examples of joints in training images; (b) Augment the dataset by reflection and rotation; (c) Extract random negative examples from images; (d) Compute features for all examples; (e) Train a soft linear support vector machine; (f) Mine hard negatives from training images and retrain the support vector machine.

a

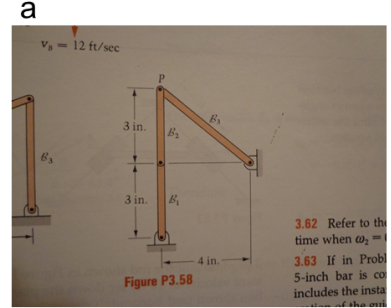

$\mathrm{b}$

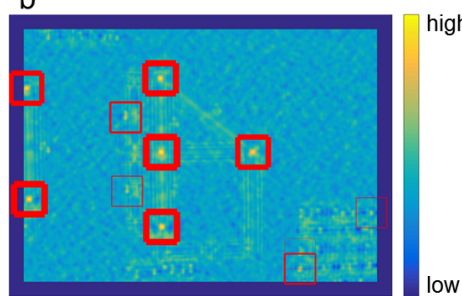

Figure 4: (a) An example image and (b) its corresponding heat map for joint detection, where color encodes distance to the decision boundary and bounding boxes highlight local maxima. Bounding box thickness positively correlates with detection confidence.

300 the SVM. This is especially critical for our problem domain be301 cause most of the background in textbook images and sketches 302 is blank, and therefore the initial set of negative training ex${ }_{303}$ amples may not accurately reflect the diversity of the negative 304 image space.

305 After training, the SVM can be used on a test image to clas306 sify the image patch centered at each pixel. The result is a 307 heat map, in which distance from the decision boundary (hyper308 plane) encodes detection confidence. In our method, we ignore 309 pixels with confidence less than zero (negative distance to the 310 hyperplane indicates the patch is more likely to not contain a 311 joint) and then apply the non-maxima suppression technique in 312 [24] to isolate local maxima. Figure 4 illustrates this concept 313 on an example image. The remaining detected joint locations 314 and associated confidence values are then passed to later stages 315 of the algorithm.

\section{5.1.1. Multiple context-based classifiers}

317 Preliminary experiments using the fixed-window SVM clas318 sifier described above revealed that text and annotations in an 319 image frequently trigger false joint detections with high con320 fidence. These false positives can be problematic for our op321 timization routine, which relies on detection confidence in the 322 computation of multiple fitness objectives (see section 5.3.2). ${ }_{323}$ With this in mind, we introduced the idea of using context cues ${ }_{324}$ from larger neighborhoods in the image to better discriminate 325 between true and false joints [21]. More specifically, we train 326 three SVM classifiers on HOG features over increasing win327 dow size and employ a weighted sum of the distances to each ${ }_{328}$ decision boundary as the indicator of detection confidence. The ${ }_{329}$ HOG descriptor parameters are modified according to window
330 size such that all image patches have the same number of fea${ }_{331}$ tures; in other words, larger window sizes yield coarser spatial $3 з 2$ binning. Unlike many multi-scale classification schemes, we do ззз not run all classifiers in parallel on the full image. Instead, we 334 implement a serial approach, in which the root classifier instan335 tiates potential joint detections and then the two context classi336 fiers adjust the confidence level of those detections accordingly. ${ }_{337}$ Refer to [21] for additional details regarding classifier weights $3 з 8$ and example results for this detection scheme.

\section{5.1.2. Foreground extraction}

340 The inclusion of multiple context classifiers has the primary ${ }_{341}$ benefit of increasing the confidence gap between true positive 342 joints and false positive joints, on average. In order to increase 343 the overall precision of the joint detection scheme, i.e. to re344 duce the number of false positives, we filter out background de345 tections using a greedy foreground extraction algorithm $\left(h_{1}\right.$ in ${ }_{346}$ Figure 2). The algorithm is depicted in Figure 5 and proceeds 347 as follows. First, a Sobel edge detector [20] is run over the satu348 rated grayscale image, yielding a set of boundaries. The bound349 aries are dilated by a fixed radius (6-8 pixels for a $600 \times 800 \mathrm{im}-$ 350 age), then connected regions are extracted. We select the region 351 with the maximum area (i.e. number of boundary pixels), but 352 unlike [21], we only count edge pixels in the middle $50 \%$ of the 353 image. This is motivated by the observation that most of the 354 foreground is typically near the center of the image, although it 355 does not restrict objects from being close to the image bound356 aries. The foreground mask corresponds to the region with the 357 highest pixel count. We determined empirically that it is use358 ful to fill the holes in the foreground region when dealing with 359 sketches, but this is not necessary for textbook graphics. This 360 approach yields $100 \%$ accuracy for the images used throughout ${ }_{361}$ this paper, meaning the filtering process never removes a true 362 joint from consideration. We hypothesize that this simple step 363 has a large impact on the quality of joint detection, which we 364 investigate further in section 6 .

\section{5.2. Detecting rigid bodies}

366 As alluded to in section 4, rigid bodies are not explicitly rec367 ognized using a sliding window detector; rather, they are iden368 tified by local features from pairs of detected joints. This de369 cision was motivated by the observation that rigid bodies ex370 hibit high variance in shape, color, texture, and size, making 371 it difficult to learn a reliable detector using typical image fea372 tures. Since any rigid body must be connected to at least two 
a

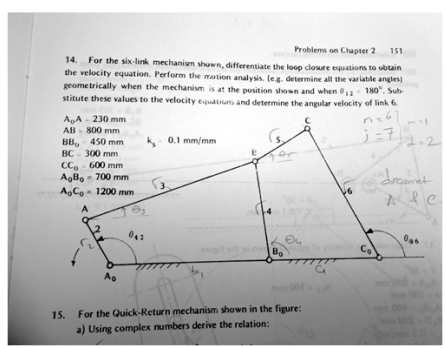

b

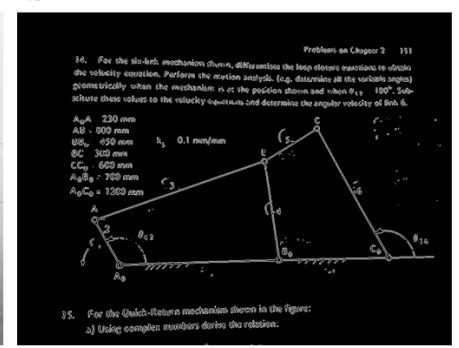

C

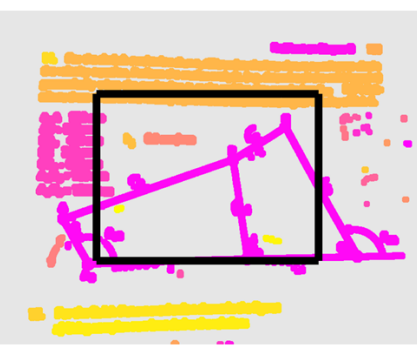

d

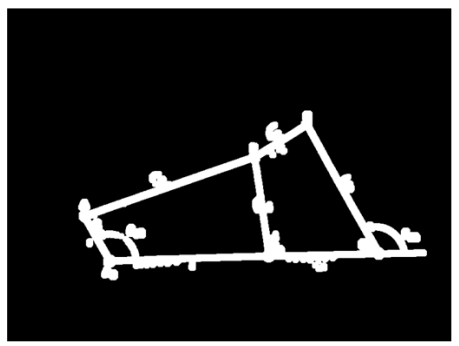

Figure 5: Greedy foreground extraction on a sample textbook image. (a) The input grayscale image after saturating the top and bottom $1 \%$ of pixels. (b) A binary mask of boundaries detected by the Sobel edge filter. (c) After dilating the boundaries by a small amount, regions are grouped together (distinguished here by different colors) and edge pixels are counted for each region. Note that only pixels inside the black bounding box are counted and the background (grey) is ignored. (d) The region with the highest count is identified as the foreground.

a

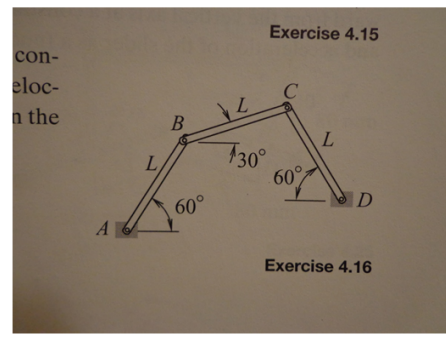

b

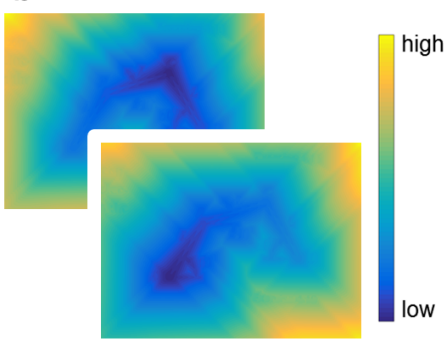

C

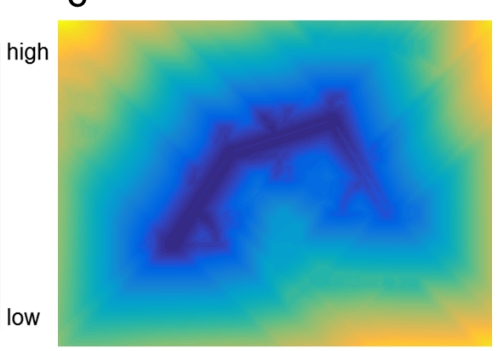

d

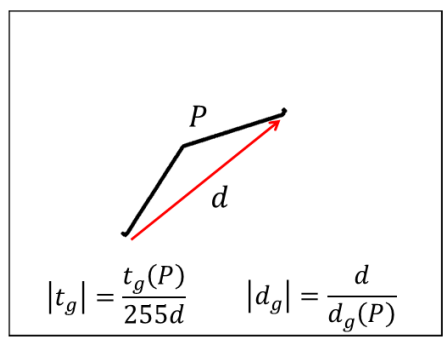

Figure 6: Computation of normalized geodesic time and distance for a pair of points in a sample image. (a) The grayscale image, from which we are comparing the pin joints at A and C. (b) Heatmaps indicate the geodesic time between pin A (front) or pin C (back) and every other pixel in the image. (c) The sum of the heatmaps can be used to extract the optimal path between the pair of points. (d) The optimal path $P$ has an associated distance (pixel count) and time (sum of the pixel intensities along the path). The distance $d$ between the pair of points is used to normalize the geodesic measures between 0 and 1 .

${ }_{373}$ joints ${ }^{3}$, we have already reduced the search space by identify374 ing probable joints and can now check pairs of joints for body 375 characteristics.

376 In contrast to our previous work in this area [21, 22], we cur377 rently present a supervised learning approach for the task of 378 body detection ( $g_{2}$ in Figure 2). Specifically, we train a soft, 379 linear SVM in the four-dimensional space defined by the fol380 lowing features: (i) normalized geodesic time; (ii) normalized ${ }_{381}$ geodesic distance; (iii) minimum joint confidence for the pair in ${ }_{382}$ question; and (iv) maximum joint confidence between the same звз joints.

${ }_{384}$ The geodesic time [59] between any pair of pixels $(p, q)$ in a 385 grayscale image is defined as

$$
t_{g}(p, q)=\min \left\{t_{g}(P) \mid P \text { links } p \text { to } q\right\}
$$

386 and the geodesic time of a given path $P$ of length $n$ connecting ${ }_{387}$ two pixels is given by

$$
t_{g}(P)=\frac{I_{p_{0}}}{2}+\frac{I_{p_{n}}}{2}+\sum_{i=1}^{n-1} I_{p_{i}}
$$

388 where $I_{p_{j}}$ is the pixel intensity of the $j$ th pixel on the path. In 389 other words, the geodesic time indicates how long it would take

\footnotetext{
${ }^{3}$ The exception to this rule is a pendulum, which contains a rigid body connected by only one pin; this case is excluded from the current domain of interest due to lack of complexity.
}

390 to traverse an image "landscape", if you followed the quickest 391 path between points and height of the landscape is determined 392 by pixel intensity. We believe this is a useful metric for char393 acterizing rigid bodies in mechanical linkages because there is 394 typically a path through dark boundary lines for joints located 395 on the same body. As a result, we can expect the geodesic time 396 for true pairwise connections to be low and for false connec397 tions to be high. To scale all data in the range of $[0,1]$, we 398 divide the geodesic time between two points by the worst-case 399 scenario, which is a line of white pixels connecting the points. ${ }_{400}$ Without this normalization, the algorithm may exhibit positive ${ }_{401}$ bias toward false connections between joints that are near each ${ }_{402}$ other and negative bias toward true connections in which the 403 joints are far apart.

${ }_{404}$ On its own, normalized geodesic time is likely insufficient for ${ }_{405}$ discriminating between true joint connections and false connec${ }_{406}$ tions. The reason for this is simple: mechanical linkages can be ${ }_{407}$ viewed as chains of connected bodies. As such, there should be 408 a path with low geodesic time between joints at opposite ends 409 of the chain that are not on the same rigid body. To combat ${ }_{410}$ this challenge, we include a second metric called normalized ${ }_{411}$ geodesic distance.

${ }_{412}$ The geodesic distance [59] between any pair of pixels $(p, q)$ ${ }_{413}$ in a grayscale image is defined as

$$
d_{g}(p, q)=\left\{L(P) \mid t_{g}(P)=t_{g}(p, q)\right\}
$$

414 where $L(P)$ is the length (number of pixels) of the minimum 


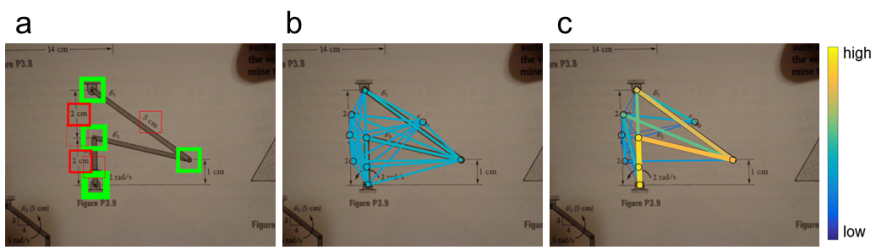

Figure 7: Comparison of rigid body detection approaches. (a) Sample image with true positive (green) and false positive (red) joint detections highlighted; (b) Previous unsupervised method based only on normalized geodesic time; (c) Current supervised approach using four features.

${ }_{415}$ path $P$ connecting $p$ and $q$. To scale all data in the range of $416[0,1]$, we apply the following normalization

$$
\left|d_{g}(p, q)\right|=\frac{\|p, q\|}{d_{g}(p, q)}
$$

${ }_{417}$ where $\|p, q\|$ is the Euclidean distance between $p$ and $q$. Based 418 on this formula, pairs of joints that have an approximately 419 straight-line minimum path will yield high values for normal420 ized geodesic distance, while pairs that require a longer, ob${ }_{421}$ scure path will result in low geodesic distance values. An il422 lustrative summary of the geodesic computations is shown in ${ }_{423}$ Figure 6.

424 While low geodesic time and high geodesic distance are good 425 indicators that a pair of joints belong to the same rigid body, 426 there are some limitations. Consider, for instance, the exam${ }_{427}$ ple depicted in Figure 7. In this case, multiple false joint de${ }_{428}$ tections are located near the edge of a rigid body (Figure 7a). ${ }_{429}$ Thus, the normalized geodesic time and distance between these 430 false joints and true joints will likely create false rigid body con${ }_{431}$ nections that are indistinguishable from true connections (Fig${ }_{432}$ ure $7 \mathrm{~b}$ ). In order to mitigate this effect, we add pairwise joint ${ }_{433}$ confidences as features for the rigid body classifier. In this way, 434 false connections may be correctly identified as such due to the 435 low relative confidence of the false joint (Figure 7c).

\section{5.2.1. Training}

${ }_{437} \quad$ Using the results of the pin joint detector as context cues for 438 rigid body detection has an important implication for training ${ }_{439}$ protocol. Given the sequential nature of the vision pipeline, the 440 rigid body detector is prone to overfit the training data if all ${ }_{441}$ samples are used at once to train the previous detector. Ideally, 442 we would like to train the rigid body detector using context cues ${ }_{443}$ that mimic joint detection behavior during testing. To accom${ }_{444}$ plish this, we implement a stacked training procedure similar to 445 [54] and described in [10, 63].

${ }_{446}$ The underlying idea of stacked learning is that training data 447 for a classifier that relies on information from a previous clas448 sifier should only be generated from images that were not used ${ }_{449}$ for training the previous classifier. In this sense, stacked learn$450 \mathrm{ing}$ is similar to cross-validation. Figure 8 demonstrates this ${ }_{451}$ concept. To produce training data for rigid body detection, we 452 split the training database into $k$ equal subsets $(k=5$ for all ${ }_{453}$ experiments in this paper). Then, we train a joint detector on ${ }_{454}(k-1)$ subsets and test it on the remaining subset. By repeat${ }_{455}$ ing this process $k$ times, we effectively create an unbiased set of

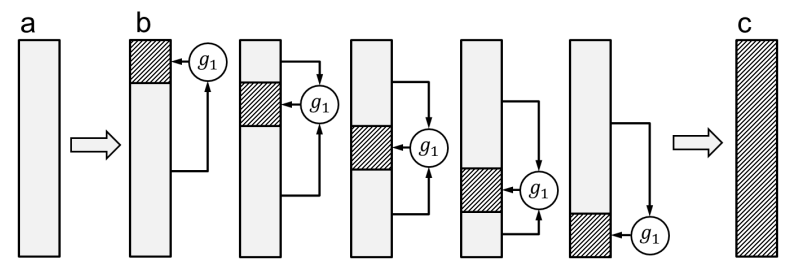

Figure 8: Stacked learning protocol. (a) The original training database is split into $k$ folds. (b) For each fold, the $g_{1}$ classifier is trained using the remaining $(k-1)$ folds. (c) The test results for each fold are compiled into a new training database for subsequent classifiers that has the same size as the original database.

456 joint detections for each image in the original training database. ${ }_{457}$ This data is subsequently used to train the rigid body detector ${ }_{458}$ using the aforementioned features.

\section{5.2.2. Filtering unused joints}

460 During testing, all pairs of detected joints in an image are ${ }_{461}$ evaluated using the learned SVM. Similar to the joint detection 462 protocol, the confidence of a rigid body connection is deter463 mined by its distance to the SVM decision boundary, and con464 nections with distance less than zero are discarded. As a result, 465 it may occur that one or more joints have no positive connec466 tions to other joints. As a rule, we remove such joints because ${ }_{467}$ they are no longer useful for the optimization routine $\left(h_{2}\right.$ in Fig468 ure 2).

\section{${ }_{469}$ 5.3. Optimizing mechanical structure}

470 Given the output from the vision pipeline, which is a set 471 of confidence values associated with detected joints and pair472 wise joint connections, the problem becomes one of constrained 473 multiobjective optimization $\left(h_{3}\right.$ in Figure 2$)$. Specifically, we 474 seek to find a hypothesis of a mechanical linkage that is strongly 475 consistent with what has been detected in the image as well as 476 reasonable in terms of kinematic simulation. To that end, we 477 employ the nondominated sorting genetic algorithm (NSGA-II) 478 introduced by Deb et al. [19]. This algorithm was selected 479 for several reasons, including its well-known success in solv480 ing real-world applications, its ability to quickly find a diverse 481 set of good solutions for multiple conflicting objectives, and the 482 unique opportunities it provides for handling constraints. No483 table details specific to the present domain are outlined below.

\section{5.3.1. Representation}

${ }_{485}$ We represent a mechanical linkage using a structured graph, 486 similar to [44], but with vertices representing joints and edges ${ }_{487}$ corresponding to pairs of joints on the same rigid body. It is 488 important to note that edges do not explicitly identify rigid bod489 ies (i.e. there is not a one-to-one correspondence); in fact, any 490 body containing more than two joints will be encoded as a fully 491 connected subgraph comprising multiple vertices and edges. In 492 addition, we relax the constraint that multiple pins fixed to the 493 ground must share an edge because a typical image may not 494 exhibit strong visual cues that grounded pins are connected. ${ }_{495}$ Consequently, the graph representation of a given mechanism 
a

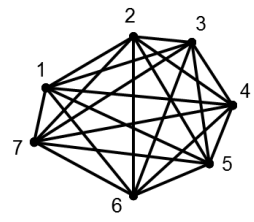

b
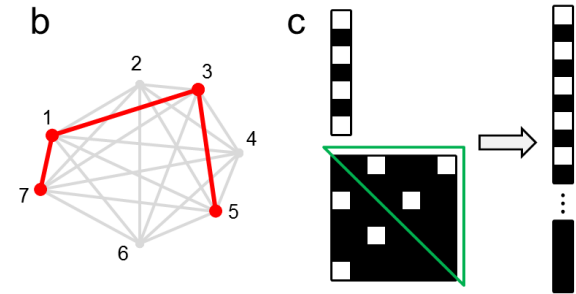

Figure 9: Phenotype-genotype mapping. (a) An artificial example of the phenotype space comprising all available graph vertices and edges. Note that the graph does not have to be fully connected as shown here. (b) A candidate solution, highlighted in red, contains a subset of the available graph. (c) The first part of the genotype encodes which vertices exist in the solution, and the second part dictates which edges should be included. The latter portion is derived from the upper triangular adjacency matrix.

496 is not necessarily unique; also, multiple linkages with different ${ }_{497}$ kinematic behavior can be represented by the same graph.

498 Figure 9 illustrates the phenotype-genotype mapping for our 499 problem. The genotype is a vectorized transformation of the 500 structured graph, and it consists of two parts: (i) a bit-string 501 of length $N$ to indicate the presence of a joint in an individual 502 hypothesis, where $N$ is the number of detected joints; and (ii) 503 a bit string of length $N(N-1) / 2$, which encodes the presence 504 of pairwise edges. The latter part is derived from the upper tri505 angular matrix of the adjacency matrix, which is symmetric. It 506 should be noted that the genotype does not explicitly include 507 any information regarding the spatial layout of detected joints. 508 Also, this representation allows an edge to exist in the absence 509 of one or more of its connecting vertices. To deal with this 510 issue, the bit-string that encodes vertices (joints) is used to vir511 tually mask "invalid" edges (connections) for the purpose of 512 fitness evaluation. However, the true value of those edges is re513 tained during crossover and mutation so that there is no internal 514 bias toward solutions with fewer edges. Similarly, this repre515 sentation allows a vertex to exist in the absence of one or more 516 of its connecting edges. We virtually mask such vertices during 517 fitness evaluation, but keep the true value during crossover and 518 mutation.

\section{5.3.2. Fitness criteria}

520 The ultimate goal of our recognition framework is to find the 521 mechanism topology graph that concurrently optimizes a series 522 of objectives using Pareto dominance. Specifically, we seek to ${ }_{523}$ maximize the image-based confidence of joints and connections 524 used in a solution, to minimize the confidence of unused image 525 information, and to maximize the likelihood that the mechani526 cal behavior is meaningful.

1. The joint likelihood $\left(f_{1}\right)$ is the average detection confidence of joints present in a given hypothesis $(H)$, or

$$
f_{1}(H)=\frac{\sum_{i=1}^{N} s_{j, i} y_{i}}{\sum_{i=1}^{N} y_{i}}
$$
where $s_{j, i}$ is the confidence of the $i$ th joint and $y_{i}$ is the (Boolean) value of the $i$ th gene.
531 2. Similarly, the joint connection likelihood $\left(f_{2}\right)$ for a given

\section{5} 536 537 538 548
549

\section{5.3.3. Mechanical constraints}

551 In this section, we discuss the design of the mechanical con552 straints on feasibility in more detail since this is fundamentally ${ }_{553}$ the most critical objective function; without it, the optimization 554 simply maximizes object detection output with no regard to the 555 domain of interest. By feasible, we mean that a solution should 556 represent a single, closed kinematic chain and that all rigid bod557 ies (with the exception of a world frame) should be capable of 558 motion. While it may be easy for an expert engineer to evalu559 ate these criteria, it is a nontrivial task to transform them into 560 mathematical constraints that can be used by the computer. Ide561 ally, a full kinematic simulation would provide the best insight 562 regarding feasibility, but this approach is too computationally ${ }_{563}$ expensive (imagine generating 10,000+ dynamic simulations 564 quickly). Instead, we found through trial-and-error that sim565 ple heuristics yield a reasonable estimate of feasibility without 566 the lag in computational speed.

567 The initial set of constraints we developed [22] include: (i) 568 the degrees of freedom (DOF) should be greater than zero, (ii) 569 each joint must have at least one connection, (iii) there must 570 be at least four rigid bodies, including the frame, and (iv) all 571 joints must be a minimum distance away from each other (e.g. 572 15-30 pixels). Even though each of these constraints is indeed 573 a requirement for a mechanical linkage to be feasible, our im574 plementation was perhaps too simplistic. For example, we used ${ }_{575}$ Gruebler's equation [60] to compute the DOF, such that

$$
D O F=3\left(n_{b}-1\right)-2 n_{l p}-n_{h p}
$$

576 where $n_{b}$ is the number of rigid bodies (including the world 577 frame), $n_{l p}$ is the number of lower kinematic pairs (i.e. rev578 olute and prismatic joints), and $n_{h p}$ is the number of higher 
a

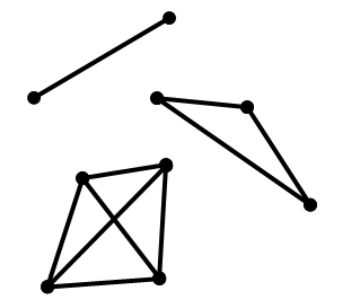

b

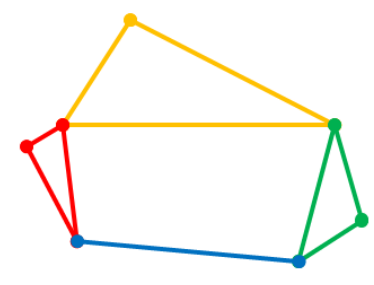

Figure 10: (a) Example cliques containing two, three, and four vertices, respectively. (b) A graph with thirteen cliques, but only four maximal cliques (distinguished by color).

579 kinematic pairs ${ }^{4}$. However, we substituted the number of edges 580 for $n_{b}$, which, as explained previously, overestimates the true ${ }_{581}$ rigid body count. This is also problematic for the third con582 straint listed above. The effect of this superficial computation 583 on the optimization results was clear during testing; many of the ${ }_{584}$ Pareto-optimal solutions were not actually sensible mechanical 585 linkages, even though they met all of the constraints we im586 posed.

587 To address this shortcoming, a significant contribution of this ${ }_{588}$ paper is the introduction of a new set of robust constraints that 589 more accurately reflect mechanical feasibility. The constraints 590 are derived from graph theory, largely from the idea of cliques 591 [31]. A clique is a fully connected subgraph - that is, all pairs 592 of vertices in the clique must have an edge between them. See ${ }_{593}$ Figure 10 for an illustration of this concept. A maximal clique 594 is one that is not contained within a larger clique. So, for exam${ }_{595}$ ple, the graph in Figure 10b has thirteen cliques, but only four 596 maximal cliques. Mechanically speaking, a fully connected set ${ }_{597}$ of links connected by pins cannot move relative to each other. 598 Therein, locating the rigid bodies in a graph amounts to find599 ing all of the maximal cliques [51]. There are three important 600 constraints related to cliques (rigid bodies). First, there must be 601 at least three maximal cliques for a feasible mechanical link602 age with only revolute joints; four rigid bodies are required ${ }^{5}$, 603 including the world frame, which may not be explicitly iden604 tified. Second, no cliques can be members of more than one 605 maximal clique (Figure 11a), for this violates the definition that 606 all pairs of joints sharing a rigid body should be connected. ${ }_{607}$ Third, no rigid bodies (maximal cliques) can form a subgraph 608 with zero DOF; the prime example of this violation is the tri609 angular graph shown in Figure 11b-c, which if you assume the 610 world frame connects the bottom two vertices, cannot move at 611 all. See Appendix A for details on the computation of these 612 three constraints. As a final constraint, because of the nature of ${ }_{613}$ kinematic chains, the graph should be connected - that is, there ${ }_{614}$ must exist a path between every pair of vertices. To determine

\footnotetext{
${ }^{4} \mathrm{~A}$ higher kinematic pair is one in which the contact between bodies is along a point or a line; lower kinematic pairs have contact along a plane. None of the mechanisms studied in this paper have higher kinematic pairs, so this last term can be ignored.

${ }^{5}$ To verify this, note that a closed kinematic chain with $m$ bodies requires at least $m$ pin joints, and then try plugging values of $m$ into $n_{b}$ and $n_{l p}$ in Equation (7). Only values $>=4$ will yield $D O F>0$.
}

a

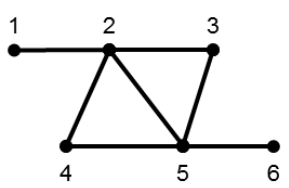

b

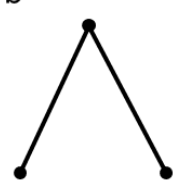

C

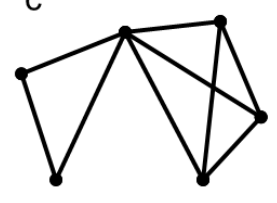

Figure 11: Mechanical constraint violations. (a) In this graph, a clique $\{2,5\}$ participates in more than one maximal clique $(\{2,3,5\},\{2,4,5\})$. (b-c) In these instances, two maximal cliques form a subgraph with zero degrees-of-freedom (i.e. if you fix the free ends, the cliques cannot move relative to each other). Note that both graphs violate the same constraint despite having different topologies.

615 whether this is true for a given solution, we use the Dulmage${ }_{616}$ Mendelsohn decomposition of the adjacency matrix [53].

To recap, the updated mechanical constraints for feasibility 618 are as follows:

1. There must be at least three maximal cliques.

2. There must be no cliques that are members of more than one maximal clique.

3. There must be no subgraphs with zero DOF.

4. There must be only one connected component.

\section{6. Experiments}

625 6.1. Data

626 For textbook graphics, we use the MECH135 dataset [22], 627 which consists of 135 images of planar mechanical linkages 628 containing only revolute joints. For hand-drawn sketches, we 629 use the MECHS250 dataset [21], which comprises 250 images 630 of sketches created by 25 engineering students ( 10 sketches per 631 user). Each sketch depicts a single planar mechanical linkage ${ }_{632}$ chosen randomly from the MECH135 dataset. All images are 633 scaled to $600 \times 800$ pixels.

\section{6.2. Methods}

${ }_{635}$ The primary goal of the experimental studies presented here 636 is to characterize the performance of each subroutine in our ${ }_{637}$ recognition framework. The three subroutines of interest are 638 joint detection (section 5.1), body detection (section 5.2), and 639 structural optimization (section 5.3). Previous work [21, 22] 640 only focused on evaluation at the optimization end, which lim${ }_{641}$ ited our ability to quantify the impact of earlier stages in the 642 pipeline when comparing algorithm modifications. In this pa${ }_{643}$ per, we systematically evaluate each stage for all combinations 644 of detections schemes and optimization constraints to deter645 mine which methods yield state-of-the-art performance and to 646 diagnose areas requiring improvement in the future. Table 1 647 summarizes the algorithm variants explored in this paper.

648 We conduct two sets of independent experimental studies, 649 one for textbook graphics and one for hand-drawn sketches. ${ }_{650}$ The former includes three textbooks for testing, and the latter 651 tests sketches from three users. Considerable effort was made to 652 select exemplary users with varied sketching style and level of ${ }_{653}$ abstraction. Unlike our previous intermodal study [21], in this 
Table 1: Description of algorithm variants

\begin{tabular}{|c|c|}
\hline \multicolumn{2}{|l|}{ Joint Detection } \\
\hline SVM & $\begin{array}{l}\text { fixed-window, soft, linear support vector machine trained on HOG } \\
\text { features }\end{array}$ \\
\hline CSVM & $\begin{array}{l}\text { cascade of three context-based support vector machines trained on } \\
\text { HOG features }\end{array}$ \\
\hline $\mathrm{SVM}+\mathrm{FG}$ & same as SVM, but with the additional foreground filter \\
\hline $\mathrm{CSVM}+\mathrm{FG}$ & same as CSVM, but with the additional foreground filter \\
\hline \multicolumn{2}{|l|}{ Body Detection } \\
\hline SVM4 & $\begin{array}{l}\text { support vector machine trained on four features including normal- } \\
\text { ized geodesic distance and time as well as confidence of detected } \\
\text { joints }\end{array}$ \\
\hline SVM2 & same as SVM4, except that joint detection confidences are ignored \\
\hline$\left|d_{g}\right|$ & normalized geodesic distance (unsupervised) \\
\hline$\left|t_{g}\right|$ & normalized geodesic time (unsupervised) \\
\hline \multicolumn{2}{|c|}{ Mechanical Constraints for Structural Optimization } \\
\hline & $\begin{array}{l}\text { NAIVE initial set based on DOF, number of joint connections, } \\
\text { and distances between joints }\end{array}$ \\
\hline GRAPH-TH & updated set based on graph theory \\
\hline
\end{tabular}

Table 2: General NSGA-II parameters

\begin{tabular}{lcc}
\hline Parameter & Symbol & Value \\
\hline \hline population size & $\mu$ & $200 N^{\dagger}$ \\
number of offspring & $\lambda$ & $\mu$ \\
maximum number of generations & $n$ & 20 \\
crossover method & - & uniform \\
crossover probability & $p_{c}$ & 0.9 \\
mutation method & - & uniform \\
mutation probability & $p_{m}$ & 0.1 \\
tournament size & $k$ & $0.02 \mu$ \\
\hline${ }^{\dagger} N$ refers to number of detected joints & &
\end{tabular}

${ }_{654}$ paper we exclusively train and test our framework on the same 655 type of image at a given time - that is, textbook graphics are 656 used to train a system that is tested on textbook graphics and ${ }_{657}$ sketches are used to train a system that is tested on sketches. ${ }_{658}$ Nonetheless, to avoid overfitting, we do not allow testing im659 ages to be involved during the training process. In fact, we do 660 not even allow images from the same subset (e.g. textbook, ${ }_{661}$ user) to be included in both training and testing. In this way, 662 we believe our protocol mimics the practical scenario of a stu${ }_{663}$ dent or engineer using a pre-trained recognition framework on 664 a new textbook or previously unseen sketches.

665 For a given experimental condition and test subset, we train 666 a joint detector using the remaining images in the correspond667 ing dataset (MECH135 or MECHS250). As needed, we train 668 a body detector using 5-fold stacked learning (section 5.2.1). ${ }_{669}$ The detectors are used to locate probable joints and pairwise ${ }_{670}$ joint connections in every test image. Then, for each image, ${ }_{671}$ NSGA-II is run five times using the general parameters listed in ${ }_{672}$ Table 2 and the appropriate mechanical constraints. We follow 673 the measures against uncertainty and post-processing methods 674 described in [21, 22]. The full implementation has been devel675 oped in MATLAB [3], and all experiments were performed on 676 an Intel(R) quad-core $3.30 \mathrm{GHz}$ CPU with 8 GB RAM.

\section{6.3. Evaluation metrics}

678 In this section, we highlight relevant performance measures 679 for each stage in our recognition framework. For joint and 680 body detection, we employ Precision-Recall (PR) curves [56]
${ }_{681}$ because they are a well-established assessment technique used 682 in computer vision [16]. For instance, PR curves are the princi${ }_{683}$ pal method for comparing algorithms in the popular PASCAL ${ }_{684}$ VOC challenge [23]. As the name implies, a PR curve is a plot 685 of precision versus recall for a binary classifier. Precision is 686 defined as

$$
\operatorname{Pr}=\frac{t p}{t p+f p}
$$

687 and recall is given by

$$
R e=\frac{t p}{t p+f n}
$$

${ }_{688}$ where $t p$ is the number of true positives (correctly identified 689 objects), $f p$ is the number of false positives (false alarms), and $690 \mathrm{fn}$ is the number of false negatives (missed objects). Precision ${ }_{691}$ reflects the number of false detections made by the classifier, or 692 the positive predictive value (i.e. How confident can we be that ${ }_{693}$ a detection is actually a good one?). Recall reflects the sensi694 tivity of the classifier to missed detections (i.e. How confident ${ }_{695}$ can we be that the classifier will not miss desired detections?). ${ }_{696}$ Both precision and recall can vary from 0 to 1 , with 1 being the ${ }_{697}$ best score. In other words, the globally optimal operating point 698 in Precision-Recall space is at $(1,1)$, or the top-right corner of 699 the plot.

700 A PR curve is generated as follows. For a set of detections, ${ }_{701}$ perform ground truth matching to locate true matches. Then, 702 sort the detections based on confidence. Vary a threshold be703 tween the minimum and maximum confidence values. At each 704 threshold, consider all detections with confidence greater than 705 the threshold to be positive detections and all others negative. 706 The relevant variables $(t p, f p, f n)$ can be computed from the ${ }_{707}$ positive/negative labels and ground truth matches, and a single 708 value for precision and recall is calculated. After computing the 709 precision and recall at each threshold, the result can be plotted.

A PR curve can provide qualitative assessment of the 711 strengths and weaknesses of a classifier. For quantitative com${ }_{712}$ parison, most researchers use the average precision (AP), or the ${ }_{713}$ area under the curve. In our experiments, the mean average pre${ }_{714}$ cision (mAP) across different textbooks or users yields a holis715 tic view of classifier performance.

716 For mechanical graph optimization, we leverage a collection 717 of different evaluation metrics to assess overall performance. ${ }_{718}$ First, we look at whether an image is solvable, meaning the true ${ }_{719}$ graph is contained within the search space of the optimization. 720 In a way, the percentage of images that are solvable reflects the ${ }_{721}$ combined quality of the joint and body detection schemes. If 722 the joint detector fails to locate one of the true joints in an im723 age, for instance, the optimization will never evolve the true so724 lution. The next metric we consider is the percentage of solved 725 images. By this we mean the number of images for which the 726 true solution was found in at least one independent run. The rel${ }_{727}$ ative difference between the percentage of solvable and solved 728 images may provide insight into the limits of the optimization. 729 An image that is solvable, but not solved, may indicate that the 730 joint and body detectors have high recall, but low mean average ${ }_{731}$ precision. In other words, weak confidence in true objects or 
a

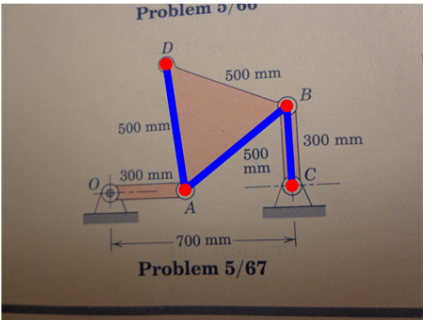

b

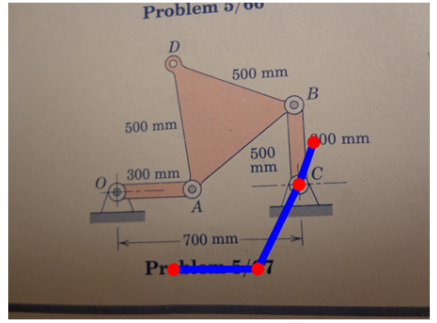

Figure 12: Both of these solutions do not match the ground truth, but are they equally bad? In (a), the solution can be corrected in one step by moving the joint at point $D$ to point $O$. The solution pictured in (b) requires multiple steps to accurately match the ground truth.

${ }_{732}$ strong confidence in false objects may restrict the ability of the ${ }_{733}$ optimization routine to evolve the true solution. Refer to [21] 734 for additional details and examples of unsolvable and unsolved 735 images.

736 Another performance measure used to assess optimization ${ }_{737}$ quality is top- $N$ accuracy. Top- $N$ accuracy refers to the percent738 age of runs in which the true solution was located in at least the ${ }_{739}$ top $N$ sorted Pareto-optimal solutions. Ideally, we desire for 740 the true solution to be the top solution (high top-1 accuracy), 741 but this may not be realistic given the fact that we have multi742 ple, conflicting objective functions. As a result, we also look 743 at top-5 accuracy and consider post hoc user selection or full 744 kinematic simulation of the top 5 solutions to be reasonable ap745 proaches for narrowing the output set of solutions.

746 In our baseline experiments [21, 22], we relied on top- $N$ ac747 curacy to measure the success of our framework. While it is a 748 valuable metric, it may be too coarse in resolution. Consider 749 the examples in Figure 12. Neither of these graphs matches the 750 ground truth, but it is clear that the graph on the left is closer 751 to the true solution than the one on the right. Is there a way to 752 capture this information? We propose a novel evaluation metric ${ }_{753}$ called the user effort ratio. The basic premise is that the worst 754 recognizer would require the user to manually create the entire 755 model on their own (similar to what current modeling software 756 requires). The best recognizer, on the other hand, would not 757 require any effort from the user for model creation. In practice, 758 our recognition framework likely exists somewhere in the mid759 dle. It may not always get the true mechanical linkage correct, 760 but it should at least give results that are close to the true solu761 tion. In this way, it is conceivable that the user could correct the 762 model with simple, intuitive strokes (e.g. add a missing joint by 763 circling it, remove a false edge by crossing it out).

764 The user effort ratio $\left(R_{E}\right)$ is thus defined as

$$
R_{E}=\frac{\text { number of steps to correct model }}{\text { number of steps for manual model creation }}
$$

765 Lower values of $R_{E}$ are preferred. The denominator is easily 766 computed as the sum of all vertices and edges in the ground 767 truth solution; the user would simply need to specify each one 768 to construct the graph manually. The numerator, however, is 769 treated a bit differently. As illustrated in Figure 13, we consider
Table 3: Average Precision for joint detection in textbook graphics

\begin{tabular}{rcccc}
\hline & SVM & CSVM & SVM+FG & CSVM+FG \\
\hline \hline Ginsberg & 0.48 & 0.79 & 0.75 & 0.88 \\
McGillKing & 0.89 & 0.88 & 0.96 & 0.95 \\
MeriamKraige & 0.91 & 0.91 & 0.92 & 0.93 \\
\hline mAP & 0.76 & 0.86 & 0.88 & 0.92
\end{tabular}

770 each of the following user operations to count as one step: (i) 771 adding a joint; (ii) adding an edge between two existing joints; 772 (iii) removing a joint and its corresponding edges; (iv) remov773 ing an edge; and (v) moving the location of a joint, keeping 774 its edges intact. This novel performance measure has two ad775 vantages. First, it provides more fine-grained information for 776 comparing optimization routines. Second, it gives an interest777 ing estimate of how beneficial our computational method will 778 be to end users - that is, how much effort will our automatic 779 recognition framework save them? In this paper, we compute 780 user effort ratio based solely on the top solution, although in 781 theory an average user effort ratio could be computed on a small 782 set (e.g. top 5 solutions).

\section{6.4. Results and discussion}

784 Sample results for both textbook graphics and hand-drawn 785 sketches are provided in Figure 14. In the following sections, 786 we discuss the results for each study separately.

\section{6.4.1. Textbook graphics}

788 The three textbooks selected for testing are referenced by au789 thor name: Ginsberg [30], McGillKing [47], and MeriamKraige 790 [48]. Each textbook contributes 3, 15, and 27 images to the 791 dataset, respectively. Precision-recall curves for joint detection 792 are shown in Figure 15 and average precision results are sum793 marized in Table 3. In general, mean average precision is high 794 ( $>0.75$ for all cases with a maximum of 0.92 for the CSVM+FG 795 case), indicating that the joint detection methods we selected 796 are largely successful. It should be noted that the results for ${ }_{797}$ Ginsberg appear noisier than the others, but this is simply due 798 to the low number of images for that textbook (3) and is not 799 representative of large-scale detection performance. Still, all 800 textbooks demonstrate the same trend of increasing mAP as 801 multiple context classifiers are added and even more so when 802 the foreground filter is applied. The markers on each plot in ${ }_{803}$ Figure 15 pinpoint the actual operating points of each classi804 fier, and they exemplify the same trend. An important outcome 805 of this assessment is that recall is always very high, meaning 806 we rarely miss a joint detection, even if there are many false 807 positives. Since it is clear that including foreground extraction 808 always yields better average precision, we only consider the $809 \mathrm{SVM}+\mathrm{FG}$ and CSVM+FG cases further in the pipeline.

810 Precision-recall curves for rigid body detection are illustrated 811 in Figure 16 and accompanied by average precision results in 812 Table 4. For this, we analyze eight experimental conditions 813 based on the two remaining joint detection schemes and the four 814 available body detection methods. The mean average precision 815 is markedly lower than the joint detection mAP (Table 3) for all 
a

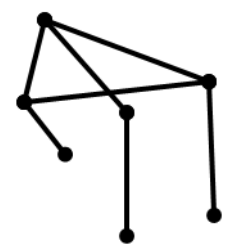

b

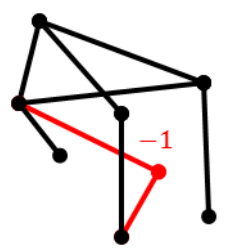

C

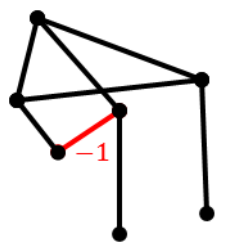

d,e

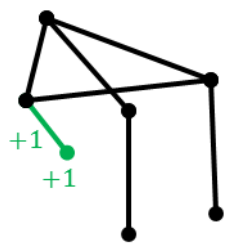

f

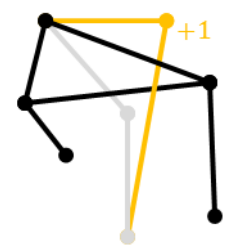

Figure 13: Artificial examples of user effort required to fix an incorrect solution. (a) The correct solution. Each of the following interactions count as one step: (b) removing a joint and its associated connections; (c) removing an edge between two joints; (d) adding a joint; (e) adding an edge between two joints; and (f) moving a joint to a new location, keeping its edges intact.
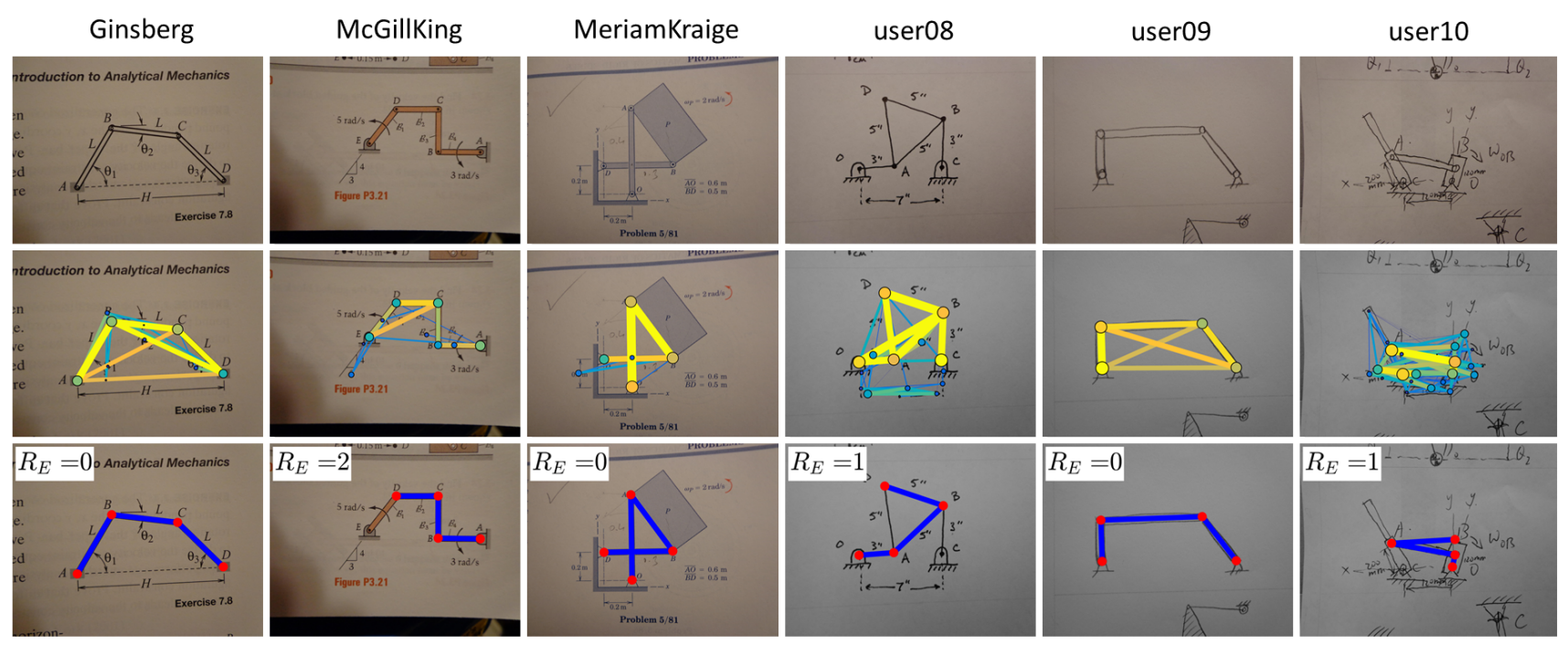

Figure 14: Sample results on textbook images and sketches. For each textbook and user, we select a representative example and show the original image (top), the output from our vision pipeline (middle), which depicts detected joints and bodies (thicker, more yellow lines and circles indicate higher confidence), and the top solution evolved during one optimization run (bottom). All solutions shown here require 2 or fewer steps for correction.

816 experimental conditions. This is most likely a result of the fact 817 that our rigid body detection space depends on the joint detec818 tor. Some false positive joints may create pairwise connections 819 with very high confidence. Also, whereas our joint detector is 820 trained on a high-dimensional (1764) feature space, the body 821 detector uses at most four features, which may not be discrimi822 native enough. Nonetheless, the results exhibit some important 823 trends. While the difference that is attributable to joint detec824 tion is small, the SVM4 method outperforms all other body de825 tection methods. Interestingly, the unsupervised approaches do 826 not fare well, and the method that is most similar to our prior 827 work (geodesic time [22]) has the lowest mAP by far. Know828 ing this, we only include the SVM4 and SVM2 conditions in 829 further analyses.

830 Figure 17 depicts the performance of our recognition frame${ }_{831}$ work at the optimization stage. The eight remaining experi${ }_{832}$ mental conditions are described in Table 5. Solvability is high ${ }_{833}(>90 \%)$ for all cases, which reemphasizes that the combined 834 vision-based detectors generate few false negatives. The per835 centage of solved images, however, varies significantly. For 836 this metric, we can draw the following conclusions: (i) the new ${ }_{837}$ graph-theoretic mechanical constraints perform better than the 8з8 old naive constraints (compare $1 \leftrightarrow 5,2 \leftrightarrow 6,3 \leftrightarrow 7,4 \leftrightarrow 8$ );
839 (ii) SVM4 leads to more solved images than SVM2 (compare $8401 \leftrightarrow 3,2 \leftrightarrow 4,5 \leftrightarrow 7,6 \leftrightarrow 8$ ); and (iii) the joint detection method that 841 works better depends on the body detection method (compare $8421 \leftrightarrow 2,3 \leftrightarrow 4,5 \leftrightarrow 6,7 \leftrightarrow 8)$. Sometimes, CSVM+FG outperforms ${ }_{843} \mathrm{SVM}+\mathrm{FG}$, but the condition with the highest overall percentage 844 of solved images uses SVM+FG (condition 5).

${ }_{845}$ There are two meaningful measures of top- $N$ accuracy, one 846 related to performance on any image and one related to perfor847 mance only on solved images. These metrics are indicated in ${ }_{848}$ Figure 17 by darker and lighter bars, respectively. One way to 849 think about this distinction is that top- $N$ accuracy on all images 850 provides a sense for the overall success of the optimization, 851 while top- $N$ accuracy on solved images represents the repeata852 bility of the optimization. For top- 1 accuracy, the naive con853 straints do better on solved images ( $99.3 \%$ for condition 1), but 854 the graph-theoretic constraints do better overall (70.2\% for con855 dition 5). Top-5 accuracy shows a similar trend, except that the 856 percentages for solved images are now relatively even among 857 all conditions.

858 Perhaps the most important takeaway of this work is the 859 user effort ratio. The results in Figure 17 are very promis860 ing. Keeping in mind that lower values indicate less re${ }_{861}$ quired effort from the user, the best experimental condition is 

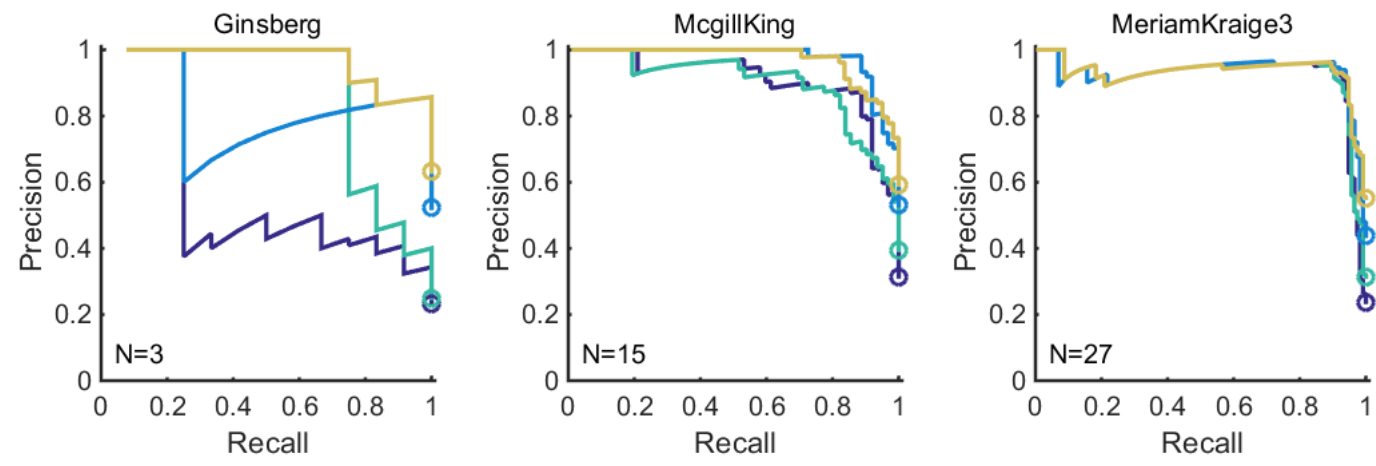

- SVM - CSVM - SVM+FG

CSVM+FG

Figure 15: Precision-Recall curves for joint detection in textbook graphics.
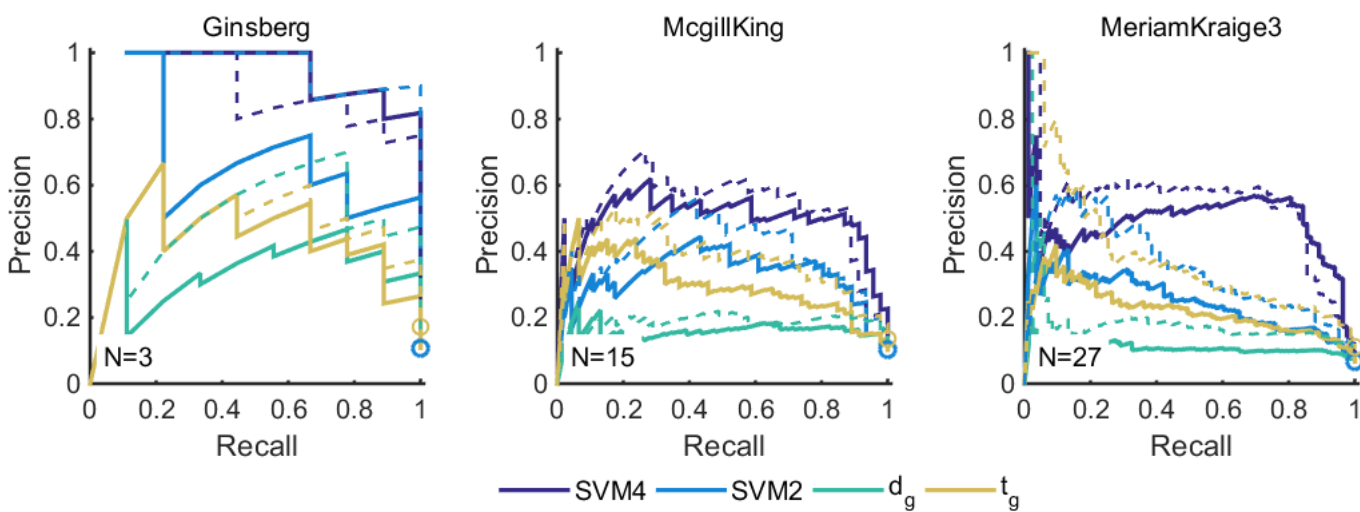

Figure 16: Precision-Recall curves for body detection in textbook graphics. Solid lines represent the SVM+FG joint detection method; dashed lines refer to CSVM+FG.

Table 4: Average Precision for body detection in textbook graphics

\begin{tabular}{|c|c|c|c|c|c|c|c|c|}
\hline \multirow{2}{*}{$\begin{array}{l}\text { Body Detection } \\
\text { Joint Detection }\end{array}$} & \multicolumn{2}{|c|}{ SVM4 } & \multicolumn{2}{|c|}{ SVM2 } & \multicolumn{2}{|c|}{$\left|\mathbf{d}_{\mathrm{g}}\right|$} & \multicolumn{2}{|c|}{$\left|\mathbf{t}_{\mathrm{g}}\right|$} \\
\hline & SVM+FG & CSVM+FG & SVM+FG & CSVM+FG & SVM+FG & CSVM+FG & SVM+FG & CSVM+FG \\
\hline Ginsberg & 0.84 & 0.78 & 0.59 & 0.85 & 0.34 & 0.49 & 0.06 & 0.10 \\
\hline McGillKing & 0.49 & 0.52 & 0.32 & 0.39 & 0.16 & 0.19 & 0.06 & 0.08 \\
\hline MeriamKraige & 0.48 & 0.53 & 0.25 & 0.34 & 0.11 & 0.18 & 0.04 & 0.06 \\
\hline mAP & 0.60 & 0.61 & 0.38 & 0.53 & 0.20 & 0.29 & 0.05 & 0.08 \\
\hline
\end{tabular}

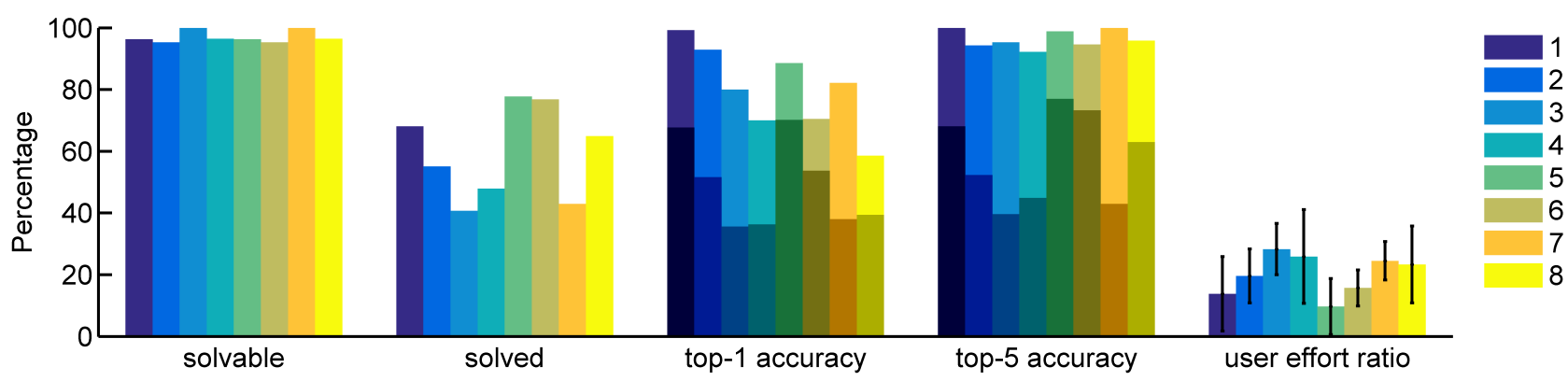

Figure 17: Optimization results on textbook graphics using eight experimental conditions. Solvable: average percentage of images from a textbook for which the optimization can find the correct solution; Solved: average percentage of images from a textbook for which the optimization does find the correct solution in at least one run; Top- $N$ accuracy: average percentage of images from a textbook for which the optimization finds the correct solution in the top $N$ solutions; darker bars account for all images, while lighter bars only include accuracy for solved images; User effort ratio: average amount of effort required by a user to correct the top solution for images from a given textbook; error bars on reveal one standard deviation. 
Table 5: Experimental conditions for optimization

\begin{tabular}{clll}
\hline ID & Joints & Bodies & Constraints \\
\hline \hline $\mathbf{1}$ & SVM+FG & SVM4 & NAIVE \\
$\mathbf{2}$ & CSVM+FG & SVM4 & NAIVE \\
$\mathbf{3}$ & SVM+FG & SVM2 & NAIVE \\
$\mathbf{4}$ & CSVM+FG & SVM2 & NAIVE \\
$\mathbf{5}$ & SVM+FG & SVM4 & GRAPH-THEORETIC \\
$\mathbf{6}$ & CSVM+FG & SVM4 & GRAPH-THEORETIC \\
$\mathbf{7}$ & SVM+FG & SVM2 & GRAPH-THEORETIC \\
$\mathbf{8}$ & CSVM+FG & SVM2 & GRAPH-THEORETIC \\
\hline
\end{tabular}

Table 6: Average Precision for joint detection in hand-drawn sketches

\begin{tabular}{ccccc}
\hline & SVM & CSVM & SVM+FG & CSVM+FG \\
\hline \hline user08 & 0.61 & 0.59 & 0.81 & 0.82 \\
user09 & 0.93 & 0.94 & 0.97 & 0.97 \\
user10 & 0.65 & 0.73 & 0.75 & 0.83 \\
\hline mAP & 0.73 & 0.75 & 0.84 & 0.87
\end{tabular}

8625 (SVM+FG/SVM4/GRAPH-THEORETIC) with a user effort ${ }_{863}$ ratio of $9.7 \%$. For all experimental conditions, our recognition 864 framework is effectively reducing user effort by at least $75 \%$.

\section{6.4.2. Hand-drawn sketches}

866 Next, we applied the same experimental conditions to im867 ages of hand-drawn sketches from three users. Figure 18 shows 868 precision-recall curves for joint detection, and Table 6 lists av${ }_{869}$ erage precision results. For any given method, the mAP is rea870 sonably high, but $6.7 \%$ lower on average than the correspond871 ing mAP for textbooks. Precision at the operating point has 872 high variance and is most likely dependent on sketching style. ${ }_{873}$ The PR curves for joint detection exemplify the same trends 874 for sketches as for textbooks (i.e. $\mathrm{CSVM}+\mathrm{FG}>\mathrm{SVM}+\mathrm{FG}>$ ${ }_{875} \mathrm{CSVM}>\mathrm{SVM}$ ).

${ }_{876}$ Precision-recall curves for rigid body detection in sketches 877 are illustrated in Figure 19 with average precision results in Ta878 ble 7. Similar to textbook graphics, mean average precision is 879 lower for bodies than for joints and the SVM4 method greatly 880 outperforms the other approaches. The CSVM+FG joint detec881 tion leads to slightly higher mAP for bodies than SVM+FG, but 882 the difference is not significant.

${ }_{883}$ Again, we only consider the eight experimental conditions 884 outlined in Table 5 for evaluating the optimization stage. The 885 results are illustrated in Figure 20. Generally speaking, the 886 recognition framework performs reasonably well on sketches, ${ }_{887}$ although not as well as textbooks. Solvability is high $(96.7 \%)$ 888 and is unaffected by choice of detection scheme or mechani$889 \mathrm{cal}$ constraints. As with textbook graphics, condition 5 is able 890 to solve the most images, but the percentage is lower (40\%). 891 Regarding top- $N$ accuracy and the user effort ratio, the dif892 ference in using SVM+FG versus CSVM+FG for joint detec893 tion appears to be small. The new mechanical constraints seem 894 to positively impact performance, but the largest improvement 895 comes from using the SVM4 method for detecting bodies over 896 the SVM2 method. One significant result from Figure 20 is that 897 conditions 5 and 6 have top-5 accuracy of $100 \%$ for solved im898 ages. In other words, even though the number of images that are
899 solved may be low, the optimization is always able to evolve the 900 correct solution in the top five candidates for images that it does 901 solve under those two conditions. The user effort required on 902 average to correct the top solution is $27-28 \%$ for all conditions 903 using SVM4 (1, 2, 5, and 6). While it may seem redundant 904 to correct an image of a paper-and-pencil sketch with digital 905 sketch-based gestures, we believe our framework may be par906 ticularly useful if both sketch creation and editing operations 907 were digital.

\section{6.4.3. Limitations}

909 There are two major limitations of this work in its current 910 state. First, the optimization operates under the assumption that 911 all true joints are at least weakly detected; in the presence of 912 a false negative, this method will never find the correct solu913 tion. Solving this issue will likely require something similar to 914 expectation-maximization, in which detected joints and bodies 915 iteratively inform the likelihood of each other so that previously 916 undetected joints can be identified if there is the strong pres917 ence of a rigid body nearby. Second, the current domain is lim918 ited in scope. We did this purposefully to ensure our problem 919 was tractable; however, future work should look to extend this 920 framework to more complex mechanical behaviors (e.g. pris${ }_{921}$ matic joints, gears, cams, intermittent contact).

\section{7. Conclusions}

923 The computational method presented here leverages well${ }_{924}$ known computer vision techniques for object recognition with 925 evolutionary methods for optimizing the graphical structure of ${ }_{926}$ planar mechanical linkages in images. We conducted a thor927 ough evaluation on textbook graphics and hand-drawn sketches 928 to demonstrate the efficacy of each stage in the framework. ${ }_{929}$ Overall performance is improved by a new supervised learn930 ing method for detecting rigid bodies that takes into account ${ }_{931}$ the context of detected joints. In addition, new constraints on ${ }_{932}$ mechanical feasibility based on graph theory ensure that Pareto93з optimal solutions are actually capable of exhibiting meaningful 934 kinematic behavior. Finally, we introduce a novel evaluation ${ }_{935}$ metric called user effort ratio that reflects the benefit derived 936 from using our automatic approach over manual model cre${ }_{937}$ ation. The results demonstrate that with very little effort $(10 \%$ ${ }_{938}$ for textbook graphics and $27 \%$ for sketches), a user can quickly ${ }_{939}$ generate kinematic models of planar mechanical linkages.

\section{Appendix A. Computing graph-theoretic mechanical con-} straints

For efficient computation of maximal cliques, we use the ${ }_{943}$ Bron-Kerbosch method with pivoting [8, 11]. The output is 944 an $m$-by- $n$ matrix $C$, where $m$ is the number of vertices (i.e. 945 joints in a mechanical linkage) and $n$ is the number of maximal ${ }_{946}$ cliques in the graph. Each column represents a maximal clique, 947 encoded as a bit-string such that

$$
C_{i j}= \begin{cases}1 & \text { if vertex } i \text { is in maximal clique } j \\ 0 & \text { otherwise }\end{cases}
$$



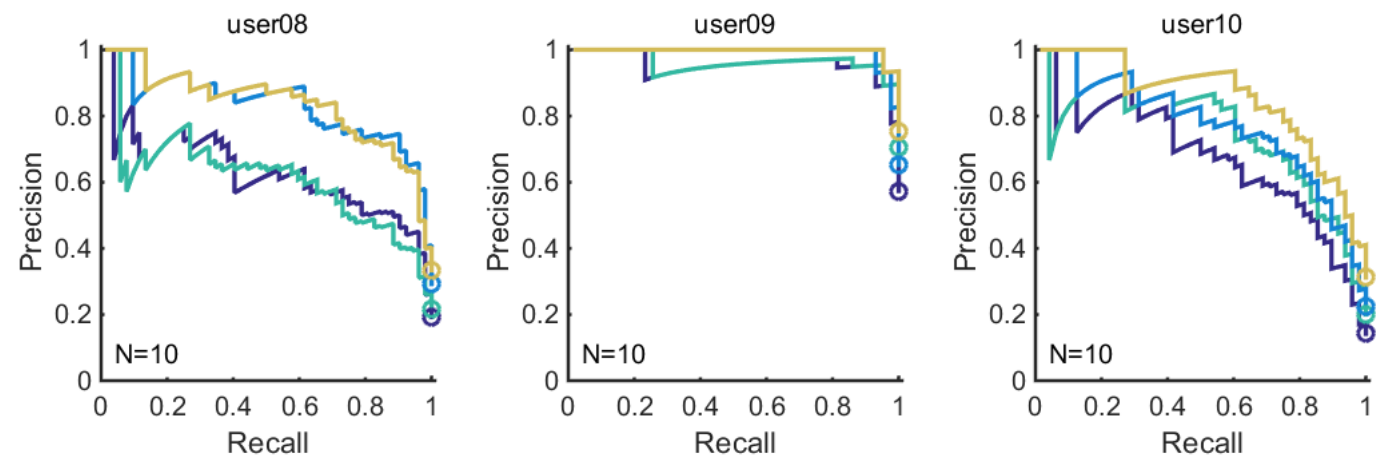

Figure 18: Precision-Recall curves for joint detection in hand-drawn sketches.
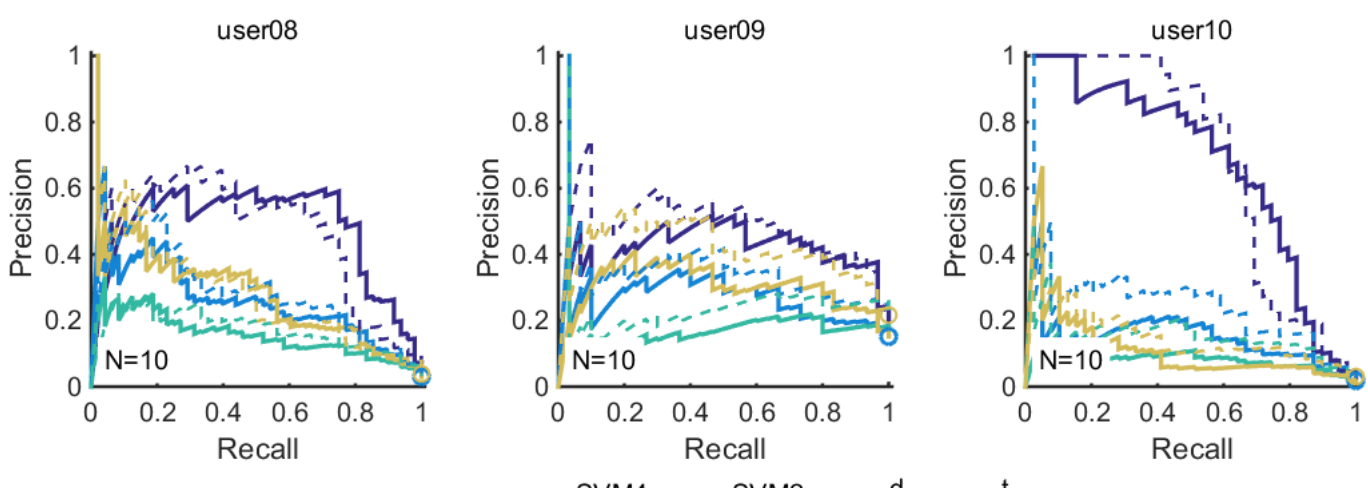

Figure 19: Precision-Recall curves for body detection in hand-drawn sketches. Solid lines represent the SVM+FG joint detection method; dashed lines refer to $\mathrm{CSVM}+\mathrm{FG}$

Table 7: Average Precision for body detection in hand-drawn sketches

\begin{tabular}{rcccccccc}
\hline $\begin{array}{c}\text { Body Detection } \\
\text { Joint Detection }\end{array}$ & \multicolumn{2}{c}{ SVM+FG } & \multicolumn{2}{c}{ SVM4 } & \multicolumn{3}{c}{$\mid$} & \multicolumn{2}{|c}{ CSVM+FG } & SVM+FG & CSVM+FG & SVM+FG & CSVM+FG & SVM+FG & CSVM+FG \\
\hline \hline user08 & 0.47 & 0.46 & 0.26 & 0.28 & 0.15 & 0.17 & 0.26 & 0.27 \\
user09 & 0.41 & 0.47 & 0.26 & 0.32 & 0.16 & 0.23 & 0.30 & 0.41 \\
user10 & 0.65 & 0.66 & 0.15 & 0.23 & 0.07 & 0.15 & 0.11 & 0.15 \\
\hline mAP & 0.51 & 0.53 & 0.22 & 0.28 & 0.13 & 0.18 & 0.22 & 0.28
\end{tabular}

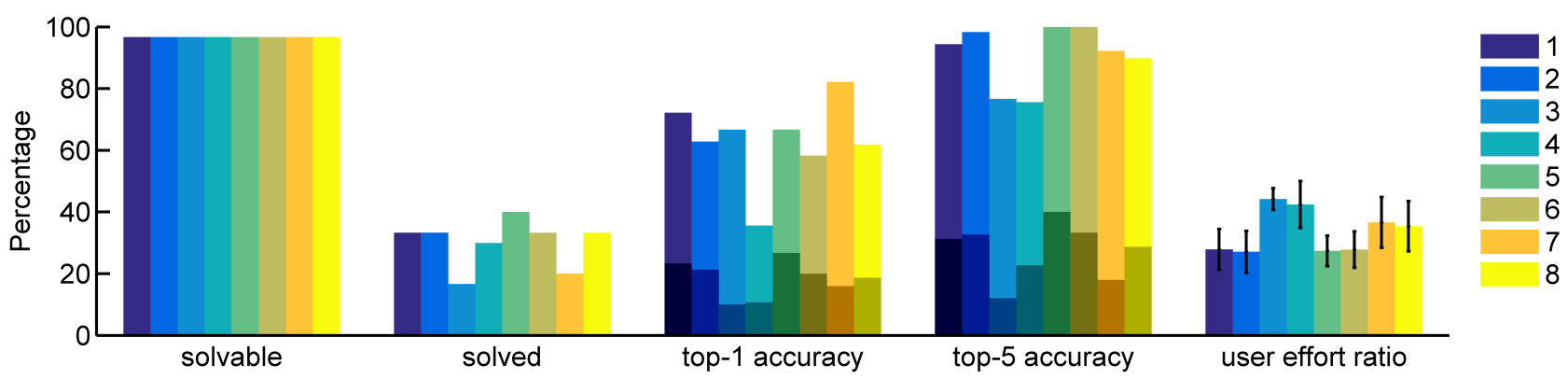

Figure 20: Optimization results on hand-drawn sketches using eight experimental conditions. Solvable: average percentage of images from a user for which the optimization can find the correct solution; Solved: average percentage of images from a user for which the optimization does find the correct solution in at least one run; Top- $N$ accuracy: average percentage of images from a user for which the optimization finds the correct solution in the top $N$ solutions; darker bars account for all images, while lighter bars only include accuracy for solved images; User effort ratio: average amount of effort required by a user to correct the top solution for images on average; error bars on reveal one standard deviation. 
948 Next, we describe how to use the matrix $C$ to compute each 98 949 of the three relevant constraints.

Next, let $B=C C^{t}$. $B$ is a symmetric, $m$-by- $m$ matrix in which $B_{i k}$ equals the number of maximal cliques containing at least vertex $i$ and vertex $k$. Taking the column-wise sum of the rows indexed by $v$ yields a vector $u$ comprising the number of vertices in $v$ that participate in a maximal clique with each of the other vertices. If any element of $u$ is greater than one, the triangular subgraph exists and the constraint is violated. . For the graph in Figure 11, it is straightforward to 980 determine that there are four maximal cliques and $C$ can be 981 written as

$$
C=\left[\begin{array}{llll}
1 & 0 & 0 & 0 \\
1 & 1 & 1 & 0 \\
0 & 1 & 0 & 0 \\
0 & 0 & 1 & 0 \\
0 & 1 & 1 & 1 \\
0 & 0 & 0 & 1
\end{array}\right]
$$

The matrix of shared vertices is then given by

$$
A=C^{t} C=\left[\begin{array}{llll}
2 & 1 & 1 & 0 \\
1 & 3 & 2 & 1 \\
1 & 2 & 3 & 1 \\
0 & 1 & 1 & 2
\end{array}\right]
$$

983 Since $A(2,3)>1$, the second and third maximal cliques (in${ }_{984}$ dexed by columns in $C$ ) share a clique and the second mechan- ${ }_{1055}$ 985 ical constraint is violated.

[2] ForceEffect Motion. Autodesk, Inc., San Rafael, California, 2014.

027 [18] J. G. De Jalon and E. Bayo. Kinematic and dynamic simulation of multi-

032 [20] P. E. Duda and R. O. Hart. Pattern Classification and Scene Analysis. John Wiley and Sons, Inc., New York, New York, 1973.

1034 [21] M. Eicholtz and L. B. Kara. Intermodal image-based recognition of pla1035 nar kinematic mechanisms. Journal of Visual Languages $\mathcal{E}$ Computing, 27:38-48, 2015.

1042 The pascal visual object classes (voc) challenge. International journal of 1043 computer vision, 88(2):303-338, 2010.

1044 [24] P. F. Felzenszwalb, R. B. Girshick, D. McAllester, and D. Ramanan. Ob1045 ject detection with discriminatively trained part-based models. Pattern 1046 Analysis and Machine Intelligence, IEEE Transactions on, 32(9):1627$1047 \quad 1645,2010$

1048 [25] P. F. Felzenszwalb and D. P. Huttenlocher. Pictorial structures for object 1049 recognition. Int J Comput Vis, 61(1):55-79, 2005.

(A.4) 1050 [26] M. A. Fischler and R. Elschlager. The representation and matching of 1051 pictorial structures. Computers, IEEE Transactions on, C-22(1):67-92, 1052 Jan 1973

053 [27] L. Fu and L. B. Kara. Recognizing network-like hand-drawn sketches: a convolutional neural network approach. In ASME 2009 International Design Engineering Technical Conferences and Computers and Information in Engineering Conference, pages 671-681. American Society of Mechanical Engineers, 2009.

[1] Adams, version 2013.2. MSC Software Corporation, Newport Beach, ${ }_{1058}^{1057}$ [28] L. Fu and L. B. Kara. From engineering diagrams to engineering models: 
1070 [33] T. Hammond and R. Davis. Tahuti: A geometrical sketch recognition sys- 1141

J. P. McCloskey. Portable trommel, Oct. 13 1998. US Patent 5,819,950.

$\begin{array}{ll}1110 & \text { D. J. McGill and W. K. King. Engineering Mechanics: An Introdu } \\ 10 \text { Dynamics. Tichenor Publishing, Bloomington, Indiana, } 2003 .\end{array}$

1111 [48] J. L. Meriam and L. G. Kraige. Engineering Mechanics, Volume 2. John 1112 Wiley and Sons, Inc., Singapore, 1993.

113 [49] D. Mundo, J.-Y. Liu, and H.-S. Yan. Optimal synthesis of cam-linkage 1114 mechanisms for precise path generation. Journal of Mechanical Design, 1115 128(6):1253-1260, 2006.

1116 [50] T. Y. Ouyang and R. Davis. A visual approach to sketched symbol recog1117 nition. In IJCAI, volume 9, pages 1463-1468, 2009.

1118 [51] S. Perera and N. Barnes. Maximal cliques based rigid body motion segmentation with a rgb-d camera. In Computer Vision-ACCV 2012, pages 120-133. Springer, 2013.

2] E. J. Peterson, T. F. Stahovich, E. Doi, and C. Alvarado. Grouping strokes into shapes in hand-drawn diagrams. In $A A A I$, volume 10, page 14, 2010.

3] A. Pothen and C.-J. Fan. Computing the block triangular form of a sparse matrix. ACM Transactions on Mathematical Software (TOMS), 16(4):303-324, 1990.

4] V. Ramakrishna, D. Munoz, M. Hebert, J. A. Bagnell, and Y. Sheikh. Pose machines: Articulated pose estimation via inference machines. In Computer Vision-ECCV 2014, pages 33-47. Springer, 2014.

5] S. Ross, D. Munoz, M. Hebert, and J. A. Bagnell. Learning message- passing inference machines for structured prediction. In Computer Vision and Pattern Recognition (CVPR), 2011 IEEE Conference on, pages 27372744. IEEE, 2011.

[56] G. Salton and M. J. McGill. Introduction to modern information retrieval. 1983.

135 [57] Y. Sato, J. Takamatsu, H. Kimura, and K. Ikeuchi. Recognition of a mechanical linkage based on occlusion-robust object tracking. In Multisensor Fusion and Integration for Intelligent Systems, MFI2003. Proceedings of IEEE International Conference on, pages 329-334. IEEE, 2003.

[58] T. Sessa. Car side window lifting mechanism, Mar. 4 1980. US Patent 4,191,060.

[59] P. Soille. Generalized geodesy via geodesic time. Pattern Recognition Letters, 15(12):1235-1240, 1994.

43 [60] E. Soylemez. Mechanisms. Middle East Technical University, Ankara, Turkey, 1993.

[61] N. Srinivas and K. Deb. Muiltiobjective optimization using nondominated sorting in genetic algorithms. Evolutionary computation, 2(3):221-248, 1994.

[62] Y. G. Woldesenbet, G. G. Yen, and B. G. Tessema. Constraint handling in multiobjective evolutionary optimization. Evolutionary Computation, IEEE Transactions on, 13(3):514-525, 2009.

[63] D. H. Wolpert. Stacked generalization. Neural networks, 5(2):241-259, 1992.

[64] Y. Yang and D. Ramanan. Articulated pose estimation with flexible mixtures-of-parts. In Computer Vision and Pattern Recognition (CVPR), 2011 IEEE Conference on, pages 1385-1392. IEEE, 2011.

[65] W. Yoo and E. Haug. Dynamics of flexible mechanical systems using vibration and static correction modes. Journal of Mechanical Design, 108(3):315-322, 1986.

[66] A. L. Yuille. Deformable templates for face recognition. Journal of Cognitive Neuroscience, 3(1):59-70, 1991.

1 [67] E. Zitzler and L. Thiele. Multiobjective evolutionary algorithms: A comparative case study and the strength pareto approach. Evolutionary Computation, IEEE Transactions on, 3(4):257-271, 1999. 

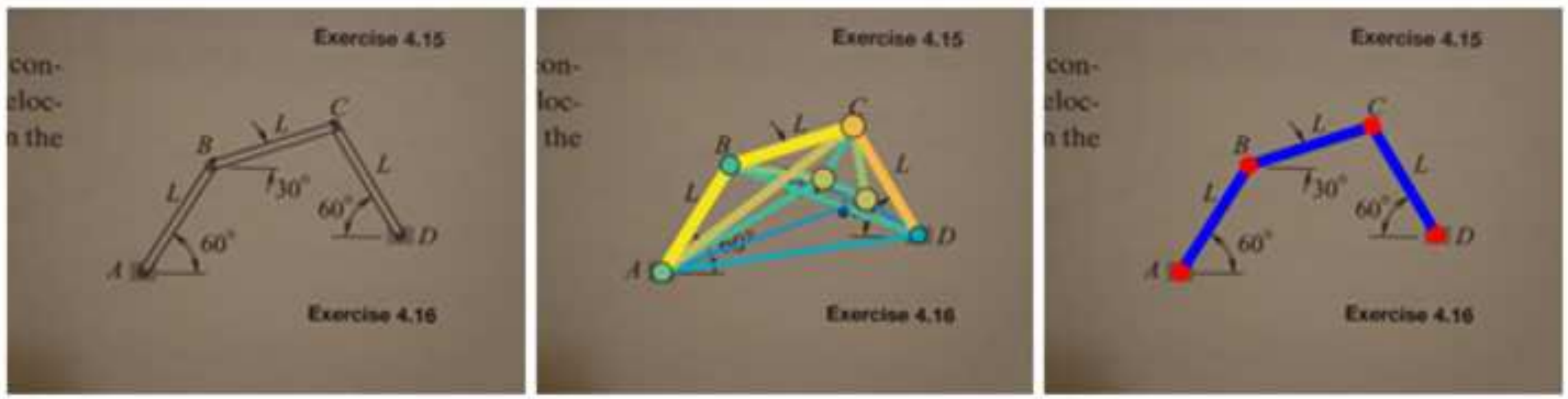